\title{
Spectral properties of finite laser-driven lattices of ultracold Rydberg atoms
}

\author{
Nikolas Tezak, ${ }^{1,2}$ Michael Mayle,, * and Peter Schmelcher ${ }^{1, \dagger}$ \\ ${ }^{1}$ Zentrum für Optische Quantentechnologien, Universität Hamburg, \\ Luruper Chaussee 149, 22761 Hamburg, Germany \\ ${ }^{2}$ Physikalisches Institut, Universität Heidelberg, Philosophenweg 12, 69120 Heidelberg, Germany
}

(Dated: May 28, 2022)

\begin{abstract}
We investigate the spectral properties of a finite laser-driven lattice of ultracold Rydberg atoms exploiting the dipole blockade effect in the frozen Rydberg gas regime. Uniform one-dimensional lattices as well as lattices with variable spacings are considered. In the case of a weak laser coupling, we find a multitude of many-body Rydberg states with well-defined excitation properties which are adiabatically accessible starting from the ground state. A comprehensive analysis of the degeneracies of the spectrum as well as of the single and pair excitations numbers of the eigenstates is performed. In the strong laser regime, analytical solutions for the pseudo-fermionic eigenmodes are derived. Perturbative energy corrections for this approximative approach are provided.

PACS numbers: $32.80 . \mathrm{Rm}, 32.80 . \mathrm{Ee}, 37.10 . \mathrm{Jk}$
\end{abstract}

\section{INTRODUCTION}

In the past decades, experimental investigations of ultracold atoms have reached an unprecedented control over the motional degree of freedom as well as the interaction of the atoms. One of the intriguing systems in this field are ultracold Rydberg atoms since they exhibit strong long-range interactions of isotropic or even anisotropic character. In fact, the interaction-induced level shift of collective states featuring multiple Rydberg excitations can be large enough to exceed the excitation laser linewidth thereby preventing the excitation of further Rydberg atoms. This dipole blockade effect was predicted by theory one decade ago along with a proposal how it could be exploited for the realization of fast twoqubit quantum gates 1, 2. Since then, a wide range of more general quantum information processing applications has emerged, putting Rydberg atoms in the focus of many theoretical as well as experimental efforts (see [3] and references therein). Experimentalists have found the dipole blockade effect 448 in gases of alkali atoms and recently for just two individually trapped atoms [9, 10. The coherence of the observed effects was demonstrated [11-14 and the resulting collective Rabi-frequency [12] has been observed.

The difficulties often encountered in working with a large number of atoms are related to the spatial structure of their gaseous samples. Because of the varying particle density typically encountered in a trap and the spatially varying intensity profile of the excitation laser, no unique collective Rabi-frequency is encountered. Several theory groups worked to circumvent these limitations by performing simulations of the excitation dynamics in

\footnotetext{
${ }^{*}$ Present address: JILA, University of Colorado and National Institut of Standards and Technology, Boulder, Colorado 80309-0440, USA

tpeter.schmelcher@physnet.uni-hamburg.de
}

unordered samples looking for signatures of the coherent dynamics [15 17] and predicting the formation of crystalline structures [18]. In [19] it was proposed to use a laser detuned from the single atom resonance to actively produce Rydberg atom pairs with the specific distance that corresponds to an interaction induced energy shift equal to the detuning. This effect was observed indirectly by measuring the interaction-induced ionization rates as a function of the interaction time [20]. In spite of these successes, it remains clear that a spatially ordered Rydberg gas is highly desirable. To this end, both theorists as well as experimentalists have concentrated on identifying a stable trapping mechanism for Rydberg states that allows a strong confinement in magnetic $21+25]$ as well as optical [26 28] traps. While the creation of lattice traps is straightforward nowadays in the optical regime, ongoing experimental effort is put into creating arrays of magnetic traps [29, 30].

First theoretical investigations of the Rydberg excitation in structured ultracold atomic gases 31 consider a ring-shaped lattice of ground state atoms, concentrating on the case where the laser coupling to the Rydberg state is weak in comparison to the next-neighbour Rydberg interaction. In the opposite regime of a dominant laser coupling, the same authors demonstrated that the system permits the formation of fermionic collective excitations [32].

In the present work, we consider a finite onedimensional lattice of ground state atoms that are coherently excited to the Rydberg state via a two-photon laser transition. We provide a thorough investigation of the spectral properties of this system for single-spaced as well as variably-spaced lattices. Different parameter regimes are covered. The model Hamiltonian that forms the basis of our investigations is derived in Sec. III In Section IIIA, the weak laser regime is analyzed where we find that a multitude of system states with well-defined excitation properties are adiabatically accessible from the ground state. Among these are the crystalline states that 
have been previously discussed in [18. In Section III B, we focus on the strong laser regime where we achieve an approximate description through the XY-model of the antiferromagnetic spin chain 33. We provide analytical solutions for the pseudo-fermionic eigenmodes as well as the perturbative energy corrections that are necessary due to the approximate nature of the description. The intermediate regime between a weak and strong laser coupling is briefly treated numerically in Sec. IIIC. In Sec. IVA we consider the case of multiple lattice spacings and describe in Sec. IVB a specific spatial setup that allows the emulation of the crystalline state transitions of a larger sized lattice. The appendix contains a derivation of the perturbative energy corrections in the strong laser regime.

\section{THE MODEL}

Starting from a quite general model including all individual atoms contributions, one can derive a simplified effective Hamiltonian that describes the system in terms of a lattice of two-level systems. To this end, the internal structure of an individual atom is described in terms of a ground state $g$, an excited (Rydberg) state $e$, and an intermediate state $m$ that we include in order to allow for an experimentally realistic excitation scheme via a two-laser setup: $g \longleftrightarrow m \longleftrightarrow e$. After transforming into a rotating frame of reference and a subsequent coarse-graining in time, an effective, static Hamiltonian is obtained and the intermediate $m$ state can be removed from the state space by an adiabatic elimination if the excitation lasers are strongly detuned with respect to this level [34, 35]. After this, the two-laser setup is modelled by an effective $g \longleftrightarrow e$ coupling and the description at each lattice site $k$ can be further reduced by employing the superatom states $|e\rangle_{k}=\left(N_{k}\right)^{-1 / 2} \sum_{i}^{N_{k}}\left|g_{1}^{(k)}, g_{2}^{(k)}, \ldots, e_{i}^{(k)}, \ldots, g_{N_{k}}^{(k)}\right\rangle$ and $|g\rangle_{k}=\left|g_{1}^{(k)}, g_{2}^{(k)}, \ldots, g_{N_{k}}^{(k)}\right\rangle$, respectively. Here $N_{k}$ is the number of atoms on site $k$. This means that either all atoms of a given site are in the ground state or they symmetrically share a single excitation [12. This reduction is justified only if the confinement to a single lattice site is sufficiently strong such that all atoms are located within a distance of each other that is smaller than the dipole blockade radius and also much smaller than the lattice constant. The symmetrization of the excited superatom state presupposes that both the effective coupling strength of the two-photon transition and its detuning from resonance are identical for all atoms within a given site. By considering solely the internal level structure of each atom, the frozen Rydberg gas regime is presumed where the atomic centre of mass motion can be neglected on the timescale of the coherent excitation dynamics.

The above considerations lead to our model Hamilto- nian

$$
H=\sum_{k=1}^{N}\left[\frac{1}{2} \Omega^{(k)} \sigma_{x}^{(k)}+\frac{1}{2} \Delta^{(k)} \sigma_{z}^{(k)}+\sum_{l=k+1}^{N} \mathcal{V}_{k l} n_{e}^{(k)} n_{e}^{(l)}\right]
$$

Here, the operators $\sigma_{i}^{(k)}, i \in\{x, y, z\}$ act on the superatom located at site $k$ and take on the usual Paulimatrix form when expressed in the local superatom basis $\mathcal{S}^{(k)}:=\left\{|e\rangle_{k},|g\rangle_{k}\right\}$. The excitation number operators may also be expressed in terms of the Pauli-operators, $n_{e}^{(k)}=\frac{1}{2}\left[\sigma_{z}^{(k)}+\mathbb{1}\right]=|e\rangle_{k}\left\langle\left. e\right|_{k}\right.$. The system is therefore formally equivalent to a spin- $1 / 2$ lattice with interactions $\mathcal{V}_{k l}$. In this picture, the contributions due to the laser are similar to the interaction of the spins with an external magnetic field with a (local) component $\Delta^{(k)}$ aligned with the spins and a perpendicular component $\Omega^{(k)}$.

Hamiltonian (1) contains three different contributions. First, the laser coupling of each single atom's ground state to the excited state is given by

$$
H_{L}=\frac{1}{2} \sum_{k=1}^{N} \Omega^{(k)} \sigma_{x}^{(k)}
$$

where $\Omega^{(k)}:=\sqrt{N_{k}} \Omega_{0}$ denotes the collective Rabi frequency for the superatom state at site $k[12$. Because of the number of atoms $N_{k}$ contributing to the excitation dynamics, $\Omega^{(k)}$ is enhanced by a factor of $\sqrt{N_{k}}$ compared to the single atom Rabi frequency $\Omega_{0}$. Second, the part which describes the site-dependent laser detuning,

$$
H_{D}=\frac{1}{2} \sum_{k=1}^{N} \Delta^{(k)} \sigma_{z}^{(k)}
$$

corresponds to an energy gap (in the effective RWA picture) of $\Delta^{(k)}$ between the excited state and the ground state of the superatom at site $k$. Finally, the Rydberg interactions read as pairwise interactions between each two sites $k, l$ with an interaction strength that depends on the spatial separation of the sites,

$$
H_{\mathrm{int}}=\sum_{k=1}^{N} \sum_{l=k+1}^{N} \mathcal{V}_{k l} n_{e}^{(k)} n_{e}^{(l)}
$$

Each summand is non-zero only if both affected sites are in the excited state. We only consider repulsive interactions, i.e., $\mathcal{V}_{k l} \geq 0$, which are common for interacting Rydberg atoms in their $n s$-state for a wide range of principal quantum numbers $n$ 36.

The contributions (2, 4 may be classified by two separate criteria: By locality, i.e., whether or not they act non-trivially on more than a single site, and by their simultaneous diagonalizability. The laser contributions, i.e., the laser coupling $H_{L}$ and the laser detuning $H_{D}$ are local, while the Rydberg interactions $H_{\text {int }}$ are by definition non-local. On the other hand, the Rydberg 
interactions and the laser detuning operator commute, $\left[H_{D}, H_{\text {int }}\right]=0$, since they can both be expressed in terms of $\sigma_{z}^{(k)}$-operators and the identity. We thus identify two interesting limiting parameter regimes: In the weak laser coupling regime the Hamiltonian is dominated by its diagonal contributions: the Rydberg interactions and the laser detuning. Alternatively, in the strong laser regime the Hamiltonian is dominated by local operators. As demonstrated in 32 for a ring lattice, this allows for an approximation of the system by an XY-model. Unless stated differently, we will restrict ourselves to global laser parameters $\left(\Omega^{(k)}, \Delta^{(k)}\right) \longrightarrow(\Omega, \Delta)$.

We describe the system in terms of the 'canonical' product basis

$$
\mathcal{S}_{N}:=\left\{\left|s_{1} s_{2} \ldots s_{N}\right\rangle, \quad s_{k} \in\{e, g\}\right\},
$$

since these states are directly accessible in experiments. Moreover, our Hamiltonian is already diagonal in this basis except for the laser coupling part $H_{L}$. Calculating the matrix elements of our Hamiltonian $H$ for the canonical product basis $\mathcal{S}_{N}$ yields a sparse matrix. Specifically, it is straightforward to show that the number of nonzero matrix elements is given by $(N+1) 2^{N}=D \log _{2} 2 D$, where $D=\# \mathcal{S}_{N}=2^{N}$ is the dimension of the state space. To simplify our notation, we define the canonical product ground state and the fully excited state as $|G\rangle:=|g g \ldots g\rangle$ and $|E\rangle:=|e e \ldots e\rangle$, respectively.

\section{SINGLE-SPACED LATTICES}

We start by considering lattices with a single lattice spacing $a$. In this case, the interaction potential is given by

$$
\mathcal{V}_{k l}=V_{|l-k|}:=\frac{C_{n}}{a^{n}|l-k|^{n}}=\frac{V_{1}}{|l-k|^{n}} .
$$

We will usually assume a Van-der-Waals interaction potential, i.e., $n=6$. The restriction to global laser parameters yields the final Hamiltonian

$$
H=\frac{1}{2} \Omega \sum_{k} \sigma_{x}^{(k)}+\frac{1}{2} \Delta \sum_{k} \sigma_{z}^{(k)}+V_{1} \sum_{l>k} \frac{n_{e}^{(k)} n_{e}^{(l)}}{|l-k|^{n}} .
$$

This Hamiltonian is invariant under reflections at the centre of the lattice. As was done for the ring lattice in 32 we designate the corresponding unitary operator for this symmetry as $\mathcal{R}$ and define it via its action on the local Pauli operators,

$$
\mathcal{R}^{\dagger} \sigma_{n}^{(k)} \mathcal{R}=\sigma_{n}^{(N-k+1)},
$$

for $n=x, y, z$. The product ground state is invariant under reflections $\mathcal{R}|G\rangle=|G\rangle$ and since the full basis can be constructed by means of the ground state and the Pauli-operators, this completely determines the form of $\mathcal{R}$. Clearly, reflecting the system twice should leave it unchanged and hence $\mathcal{R}^{\dagger}=\mathcal{R}^{-1}=\mathcal{R}$. The eigenvalues of $\mathcal{R}$ are thus given by \pm 1 .

For a weak laser coupling $|\Omega| \ll V_{1},|\Delta|$, the diagonal contributions to the Hamiltonian dominate. In this case the laser coupling leads to a small off-diagonal perturbation. Alternatively, in the case of weak Rydberg interactions the Hamiltonian can be mapped approximately to an XY-model Hamiltonian. In the following, both limiting regimes are discussed in detail and analytical formulas describing the excitation spectra are derived. For the intermediate regime, where neither of the above conditions is fulfilled, numerical simulations reveal the full spectrum.

\section{A. Weak Laser Regime}

We start by considering the weak laser regime, i.e., assuming $|\Omega| \ll V_{1},|\Delta|$. The Hamiltonian (7) is conveniently divided into two parts, $H=H_{0}+H^{\prime}$, grouping together the laser detuning with the next-neighbour Rydberg interactions to give the dominant contribution

$$
H_{0}=\frac{1}{2} \Delta \sum_{k} \sigma_{z}^{(k)}+V_{1} \sum_{k=1}^{N-1} n_{e}^{(k)} n_{e}^{(k+1)},
$$

while the perturbation consists of the laser coupling as well as the long range Rydberg interactions,

$$
H^{\prime}=\frac{1}{2} \Omega \sum_{k} \sigma_{x}^{(k)}+\frac{V_{1}}{2^{n}} \sum_{d=2}^{N-1} \frac{1}{(d / 2)^{n}} \sum_{k=1}^{N-d} n_{e}^{(k)} n_{e}^{(k+d)},
$$

which we have rewritten as a sum of contributions for a given separation $d$. Introducing the operators for the total excitation number,

$$
N_{e}=\sum_{k=1}^{N} n_{e}^{(k)}=\frac{1}{2} \sum_{k=1}^{N} \sigma_{z}^{(k)}+\frac{N}{2},
$$

and the next-neighbour excitation pair number,

$$
N_{e e}=\sum_{k=1}^{N-1} n_{e}^{(k)} n_{e}^{(k+1)},
$$

we can rewrite the unperturbed Hamiltonian as $H_{0}=$ $\Delta\left(N_{e}-N / 2\right)+V_{1} N_{e e}$. For a given state $|S\rangle=$ $\left|s_{1} s_{2} \ldots s_{N}\right\rangle \in \mathcal{S}_{N}$ from the canonical product basis [cf. [5p], the unperturbed energy eigenvalue is thus given by

$$
E(S)=\Delta\left[N_{e}(S)-N / 2\right]+V_{1} N_{e e}(S),
$$

where $N_{e}(S)$ and $N_{e e}(S)$ denote the eigenvalues of the operators $N_{e}$ and $N_{e e}$ for the state $|S\rangle$. They can readily be obtained by counting the number of excitations $\ldots e . .$. and excitation bonds ...ee... present in the sequence $S=s_{1} s_{2} \ldots s_{N}$. There are several important observations to be made at this point: 
1. $H_{0}$ is linear in $N_{e}$ and $N_{e e}$. Since $N_{e}$ and $N_{e e}$ are diagonal, this linear energy relation holds for their eigenvalues as well.

2. In general, there are multiple canonical product states of equal $\left(N_{e}, N_{e e}\right)$ which are consequently always degenerate with respect to the unperturbed Hamiltonian.

3. Depending on the specific ratio of $\Delta$ and $V_{1}$, the simple form of 12 already suggests that additional degeneracies between states of different $\left(N_{e}, N_{e e}\right)$ are possible. Since degeneracies in the RWA picture correspond to resonant laser couplings in the nonrotating frame, our result simply states that we can tune the laser to resonantly excite multi-particle states, as one would intuitively expect.

We define $D_{N}\left(N_{e}, N_{e e}\right)$ to be the dimension of each $\left(N_{e}, N_{e e}\right)$-subspace $\mathcal{H}_{\left(N_{e}, N_{e e}\right)}$. The calculation of $D_{N}\left(N_{e}, N_{e e}\right)$ is possible through a combinatorial analysis. First, note that for a given state $|S\rangle=\left|s_{1} s_{2} \ldots s_{N}\right\rangle$ the number of next-neighbour excitation pairs $N_{e e}(S)$ is fully determined by the total number of excitations $N_{e}(S)$ and the number of excited domains $e e \ldots e$ within $|S\rangle$ which we denote by $d_{e}(S)$. Whenever $d_{e}(S)$ is equal to one, $N_{e e}$ takes on its maximal value $\left.N_{e e}\right|_{d_{e}=1}=N_{e}-1$. For each additional domain $N_{e e}$ decreases by one if $N_{e}$ remains fixed. Hence, the following relation holds for any $N_{e}, N_{e e}$ :

$$
d_{e}(S)=N_{e}(S)-N_{e e}(S) .
$$

We can now calculate $D_{N}$ by analyzing the number of possibilities of how to construct appropriate sequences ' $s_{1} s_{2} \ldots s_{N}$ '. For $N_{e}=0$ we can only have $|S\rangle=$ $|g g \ldots g\rangle=|G\rangle$, hence we assume $N_{e}, d_{e} \geq 1$. We must distribute $N_{e}$ excitations across $d_{e} \leq N_{e}$ domains which leads to a factor of $\left(\begin{array}{c}N_{e}-1 \\ d_{e}-1\end{array}\right)=\left(\begin{array}{c}N_{e}-1 \\ N_{e}-N_{e e}-1\end{array}\right)$. Now, for any such division of the excited states into domains, we must count the number of ways how to distribute these domains of excited atoms across the lattice such that there is always at least one ground level site between two excited domains. The number of ground level sites is given by $N_{g}=N-N_{e}$. The domains of excited sites can thus be inserted at $N_{g}-1$ positions between two ' $g$ '-characters or at the two positions at either end of the lattice. Hence, there are $N_{g}+1$ positions across which we distribute $d_{e}$ excited domains and we must multiply the above result by a factor of $\left(\begin{array}{c}N_{g}+1 \\ d_{e}\end{array}\right)=\left(\begin{array}{c}N-N_{e}+1 \\ N_{e}-N_{e e}\end{array}\right)$. Together, we find

$$
D_{N}\left(N_{e} \geq 1, N_{e e}\right)=\left(\begin{array}{c}
N_{e}-1 \\
N_{e}-N_{e e}-1
\end{array}\right)\left(\begin{array}{c}
N-N_{e}+1 \\
N_{e}-N_{e e}
\end{array}\right)
$$

and $D_{N}\left(0, N_{e e}\right)=\delta_{N_{e e}, 0}$.

The spectrum of Hamiltonian (8), i.e., the full set of unperturbed energy eigenvalues as a function of a varying ratio $\Delta / V_{1}$ exhibits points of high degeneracy, see figure 1. These occur only at specific rational values of

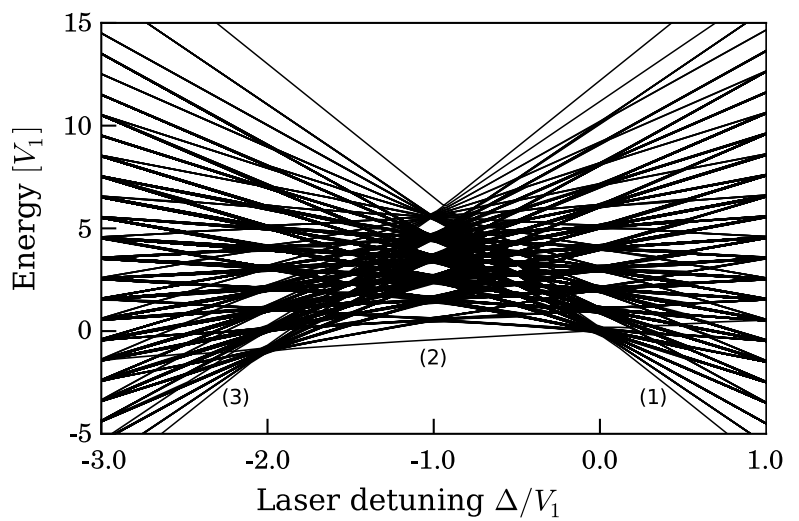

FIG. 1: Energy spectrum for $N=13$ lattice sites and a constant laser coupling $\Omega=0.05 V_{1}$. At specific rational values of $\Delta / V_{1}$ many energy levels are almost degenerate. Depending on $\Delta / V_{1}$ the energetic ground state in the RWA frame is given by (1) the canonical product ground state, (2) the alternating state $|e g e \ldots g e\rangle$, and (3) the fully excited state.

$\Delta / V_{1}$. In principle, these can be calculated from 12 if one takes into consideration the combinatorially possible combinations of $N_{e}$ and $N_{e e}$. For $\Delta=0$ the lasers are tuned to atomic resonance. In this case the state $|g g \ldots g\rangle$ is degenerate (within the effective RWA picture) with all states that lack neighbouring excitations which corresponds to dipole-blocleqed states. However, for nonzero detuning the state $|g g \ldots g\rangle$ can also be brought to degeneracy with other states. Physically, this corresponds to a situation in which the lasers resonantly couple $|g g \ldots g\rangle$ to states containing pairs of neighbouring excitations. Note, however, that due to the local nature of the laser coupling Hamiltonian $H_{L}$, these couplings require the presence of intermediate, in general off-resonant states. A state from a given $\left(N_{e}, N_{e e}\right)$ subspace can be coupled via $H_{L}$ to states from $\left(N_{e} \pm 1, N_{e e}\right)$, $\left(N_{e} \pm 1, N_{e e} \pm 1\right)$ and $\left(N_{e} \pm 1, N_{e e} \pm 2\right)$.

A particular example is provided by $\Delta / V_{1}=$ $-1 / 2$ : In this case $|g g \ldots g\rangle$ is degenerate with states containing exactly two neighbouring excitations $\{|e e g \ldots g\rangle, \mid$ geeg $\ldots g\rangle, \ldots|g \ldots g e e\rangle\}$. The general condition for a degeneracy between two canonical product states $\left|S_{1}\right\rangle$ and $\left|S_{2}\right\rangle$ is given by

$$
\frac{\Delta}{V_{1}}=-\frac{N_{e e}\left(S_{1}\right)-N_{e e}\left(S_{2}\right)}{N_{e}\left(S_{1}\right)-N_{e}\left(S_{2}\right)} .
$$

For $N_{e}\left(S_{1}\right) \neq N_{e}\left(S_{2}\right)$ and $N_{e e}\left(S_{1}\right) \neq N_{e e}\left(S_{2}\right)$ the equation can only be solved if the ratio $\Delta / V_{1}$ is rational because the eigenvalues of $N_{e}$ and $N_{e e}$ are integral. A combinatorial analysis reveals that the quantum numbers $N_{e}$ and $N_{e e}$ obey the following constraints in addition to being integral:

$$
\begin{aligned}
N_{e}=0 & \Rightarrow N_{e e}=0 \\
1 \leq N_{e} \leq\lceil N / 2\rceil & \Rightarrow 0 \leq N_{e e} \leq N_{e}-1 \\
\lceil N / 2\rceil<N_{e} & \Rightarrow 2 N_{e}-N-1 \leq N_{e e} \leq N_{e}-1 .
\end{aligned}
$$


These render it difficult to provide a concise, general quantitative analysis of the possible degeneracies.

However, to gain a better understanding of the spectrum and its degeneracies we can visualize the combinatorial possibilities for $\left(N_{e}, N_{e e}\right)$ and try to recognize general principles that are independent of the lattice size. To this end, figure 2 illustrates all possible pairs $\left(N_{e}, N_{e e}\right)$ for $N=13$. For a fixed ratio $\Delta / V_{1}, \sqrt{12}$ can also be interpreted as describing the lines of constant energy in the $\left(N_{e}, N_{e e}\right)$ plane. These lines can be constructed by drawing a line of slope $\lambda=-\Delta / V_{1}$ through each valid $\left(N_{e}, N_{e e}\right)$ point. A degeneracy occurs whenever two or more points lie on the same line. The number $\xi$ of remaining non-degenerate energy levels depends sensitively on the ratio $\Delta / V_{1}$. The minimal such number $\xi_{\min }$, i.e., the highest degeneracy is attained for $\Delta / V_{1}=-1$. This is true independently of $N$ since it follows from the shape of the distribution of possible $\left(N_{e}, N_{e e}\right)$ pairs. However, the actual value of $\xi_{\min }$ depends on the system size and is given by $\xi=\lceil N / 2\rceil+1$. The maximal value of $\xi$, on the other hand, is determined by the possible $\left(N_{e}, N_{e e}\right)$ pairs for a fixed number of lattice sites and reads $\xi_{\max }=1+\lceil N / 2\rceil^{2}$. Results of a systematic calculation of the number of non-degenerate energy levels for many different ratios $\Delta / V_{1}$ are shown in Figure 3 for $N=13$. When moving away from the point of the highest level of degeneracy $\left(\Delta / V_{1}=-1\right)$ the number of non-degenerate energy levels increases from its minimal value $\xi_{\min }=8$ and rapidly reaches its maximal value $\xi_{\max }=50$, except for the points of integer $\Delta / V_{1}$ for which pronounced local minima of $\xi$ are encountered.

In the following, we proceed by analyzing the most relevant parameter regimes in more detail. We are especially interested in the level structure of the energy band that includes the canonical product ground state $|G\rangle$ or the fully excited state $|E\rangle$. For a strongly blue detuned laser $\Delta / V_{1} \approx-2$, for example, we find that the fully excited state $|E\rangle$ is degenerate with all other states that fulfil $N_{e e}=2 N_{e}-N-1 \geq 0$; the latter are given by the right hand side of the red 'triangle' of states compatible with the constraints, cf. figure 2, These states minimize the next-neighbour Rydberg interaction energy for a given number of excitations $N_{e}$. Another interesting parameter setting is given by $\Delta / V_{1} \approx-1 / 2$. This leads to the resonant creation of isolated pairs of neighbouring excitations. Specifically, the subspace that includes the product ground state $|G\rangle$ is characterized by the relation $2 N_{e e}=N_{e}$.

\section{The Full Blockade Regime}

The full blockade regime is attained by restricting both the laser detuning and the laser coupling to be very small compared to the next-neighbour Rydberg interaction energy. The relevant subspace that includes the canonical product ground state is then approximately (neglecting the mixing due to a non-zero $\Omega$ ) given by the 0 -eigenspace of $N_{e e}$, i.e., all states that contain no neighbouring excitations as given by the lowest row in figure 2. Note that this implies $N_{e} \leq\lceil N / 2\rceil$.

For non-zero detuning, the full-blockade subspace is energetically separated from the remaining state space if the detuning fulfils $|\Delta|<V_{1} / N$. Introducing the generalized excitation pair number operators

$$
N_{e e}^{[l]}=\sum_{k=1}^{N-l} n_{e}^{(k)} n_{e}^{(k+l)},
$$

we may rewrite our full Hamiltonian as

$$
H=\frac{\Delta}{2} \sum_{k=1}^{N} \sigma_{z}^{(k)}+\frac{\Omega}{2} \sum_{k=1}^{N} \sigma_{x}^{(k)}+V_{1} \sum_{l=1}^{N-1} \frac{1}{l^{n}} N_{e e}^{[l]} .
$$

For a positive detuning $\Delta>0$, the canonical product ground state is clearly also the ground state of the diagonal part of Hamiltonian (20). However, at $\Delta=0$, even when considering the part of the Rydberg interactions beyond nearest neighbours, this state becomes degenerate with all $\left(N_{e}=1, N_{e e}=0\right)$ states. For small negative, i.e., blue detuning, these states form a degenerate ground state energy level, up until $\Delta / V_{1}=1 /(N-1)^{n}$ at which point they cross with the $N_{e}=2$ state $|e g \ldots g e\rangle$. Further decreasing the detuning leads to a succession of ground states with an increasing number of excitations $2 \leq N_{e} \leq$ $\lceil N / 2\rceil$. In general the ground state energy level is degenerate, except when $(N-1) /\left(N_{e}-1\right)=l_{\min } \geq 2$ is integral: In this case, it is possible to evenly distribute the excitations across the lattice. hence, there exists a unique minimal energy crystal state $\left|\left[N_{e}\right]\right\rangle:=|e g \ldots g e g \ldots g e\rangle$ where the spacing between neighbouring excitations is given by $l_{\min }$. This state's eigenvalue for the generalized excitation pair operators is given by

$$
N_{e e}^{[l]}\left|\left[N_{e}\right]\right\rangle= \begin{cases}\left(N_{e}-q\right)\left|\left[N_{e}\right]\right\rangle & \text { for } q=l / l_{\min } \in \mathbb{N} \\ 0 & \text { otherwise. }\end{cases}
$$

The corresponding diagonal matrix elements of the Hamiltonian accordingly read

$$
\begin{aligned}
\left\langle\left[N_{e}\right]|H|\left[N_{e}\right]\right\rangle_{d} & =\Delta\left(N_{e}-N / 2\right)+\frac{V_{1}}{l_{\min }^{n}} \sum_{q=1}^{N_{e}-1} \frac{N_{e}-q}{q^{n}} \\
& \approx \Delta\left(N_{e}-N / 2\right)+\frac{V_{1}}{l_{\min }^{n}}\left(N_{e}-1\right) \\
& =\Delta\left(N_{e}-N / 2\right)+\frac{\left(N_{e}-1\right)^{n+1}}{(N-1)^{n}} V_{1} .
\end{aligned}
$$

When $(N-1) /\left(N_{e}-1\right)$ is non-integral, the minimal energy configurations are realized by states with excitations separated by at least two different spacings. This case is more complex to analyze in full generality. 

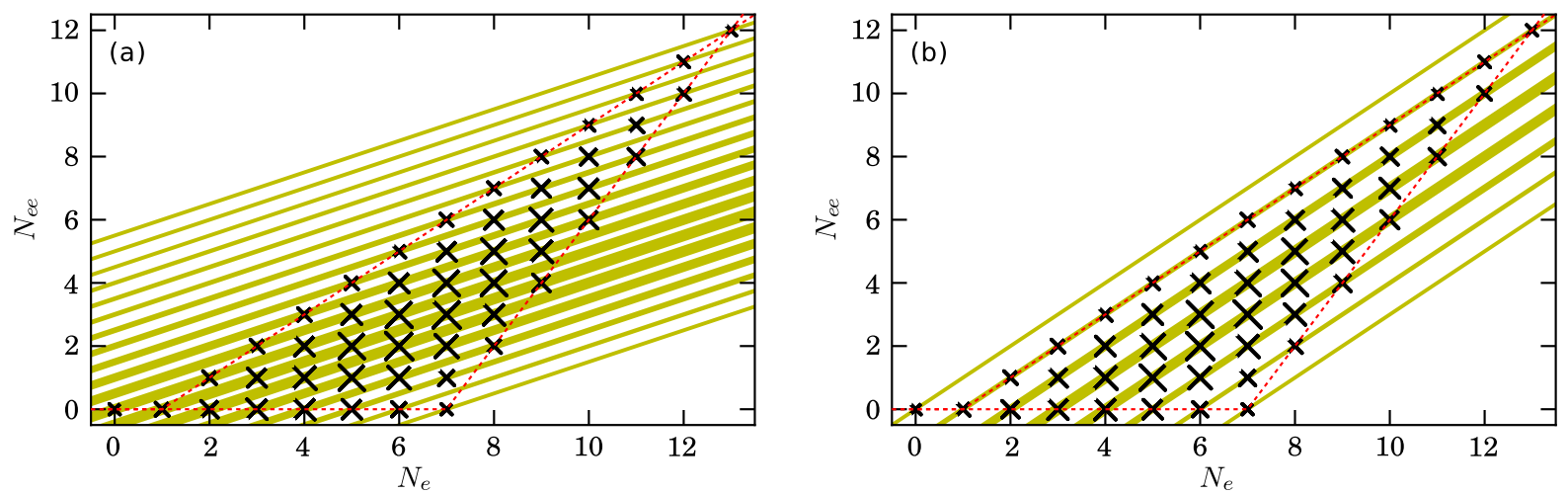

FIG. 2: Lines of constant energy (yellow) in the $\left(N_{e}, N_{e e}\right)$ plane for $N=13$ and for different slopes (a) $\Delta / V_{1}=-1 / 2$ and (b) $\Delta / V_{1}=-1$, respectively. The line thickness indicates the dimension of the associated energy eigenspace. The dashed red lines illustrate the constraints [see $\left.\begin{array}{lllll}16 & 17 & 18\end{array}\right]$. The size of an $X$-marker indicates the dimension $D_{N}\left(N_{e}, N_{e e}\right)$ of the corresponding subspace. The minimal number of non-degenerate energy levels is attained for $\Delta=-V_{1}$, cf. subfigure (b). Note that in this case the canonical product ground state $\left(N_{e}=0, N_{e e}=0\right)$ is energetically isolated.

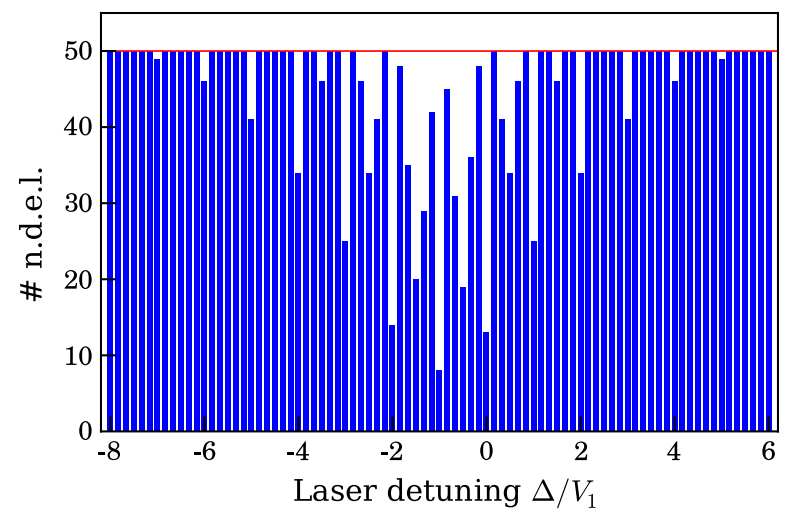

FIG. 3: The number $\xi$ of non-degenerate energy levels for different ratios $\frac{\Delta}{V_{1}}$ for $N=13$. For any $N$, the minimum number is attained for $\Delta=-V_{1}$. In our particular case we find $\xi_{\min }=8$ and $\xi_{\max }=50$, respectively.

If both $N_{e}, N_{e}-1 \in \mathbb{N}_{\geq 2}$ are divisors of $N-1$, the crystal states $\left|\left[N_{e}+1\right]\right\rangle,\left|\left[\bar{N}_{e}\right]\right\rangle$ feature an avoided levelcrossing for a well-defined detuning $\Delta_{N_{e}}^{N_{e}+1}$ :

$$
\begin{aligned}
-\frac{\Delta_{N_{e}}^{N_{e}+1}}{V_{1}}= & \left(\frac{N_{e}}{N-1}\right)^{n}\left\{\sum_{k=1}^{N_{e}} \frac{1}{k^{n}}\right. \\
& \left.+\left[1-\left(1-\frac{1}{N_{e}}\right)^{n}\right] \sum_{k=1}^{N_{e}-1} \frac{N_{e}-k}{k^{n}}+\frac{1}{N_{e}^{n}}\right\} . \\
\approx & \frac{N_{e}^{n+1}-\left(N_{e}-1\right)^{n+1}}{(N-1)^{n}} .
\end{aligned}
$$

In the thermodynamic limit $N \rightarrow \infty, N_{e} / N \rightarrow$ $f \in(0,1 / 2]$ of the exact expression 25 the results of Weimer et al. are reproduced, $-\tilde{\Delta} / V_{1} \rightarrow$ $f^{n}\left[(n+1) \zeta(n)+O\left(1 / N_{e}\right)\right][37$. For our modest lattice sizes this limit is not properly realized, but we can see that the scaling of the transition detunings with the ex- citation fraction $f=N_{e} / N$ is determined by the scaling of the interaction potential with the distance. For van der Waals interactions (i.e., $n=6$ ) this implies that the transition detunings for small excitation fractions are very small.

Whenever one is interested in the crystal states $\left|\left[N_{e}\right]\right\rangle$, it is favourable to choose a lattice size $N$ for which many different $\left|\left[N_{e}\right]\right\rangle \rightarrow\left|\left[N_{e}+1\right]\right\rangle$ transitions can be realized. For example, one can verify that $N=13$ allows for the transitions $|[2]\rangle \rightarrow|[3]\rangle \rightarrow|[4]\rangle \rightarrow|[5]\rangle$. The next candidate of an ideal lattice size is given by $N=61$ which allows for the additional transitions $|[5]\rangle \rightarrow|[6]\rangle \rightarrow|[7]\rangle$. In Section IV] we present an alternative approach how to realize these transitions by means of a non-uniform lattice with a specific pattern of lattice spacings. Figure 4 illustrates the succession of crystal states for the abovementioned case of $N=13$. An interesting aspect about this phenomenon is that although the locations of the level crossings, i.e., the specific values of the detuning at which they occur, are determined by the scaling of the interaction potential with the distance, the existence of such crystal ground states already follows from any kind of long-ranged repulsive interactions.

\section{Fully Excited Lattices}

We conclude our discussion of the weak laser regime by considering a strongly blue detuned laser $\Delta / V_{1} \approx-2$. In this case, we find that the fully excited state $|E\rangle$ is degenerate with all other states that fulfil $N_{e e}=2 N_{e}-$ $N-1 \geq 0$. In Figure 2 these states make up the right hand edge of the triangle of states compatible with the constraints.

As we have seen in figure 1 for a strongly blue detuned laser, $\Delta \leq-2 V_{1}$, the fully excited state $|e e \ldots e\rangle$ actually becomes energetically favoured in the RWA frame. Because of the long-ranged Rydberg-Rydberg interactions 


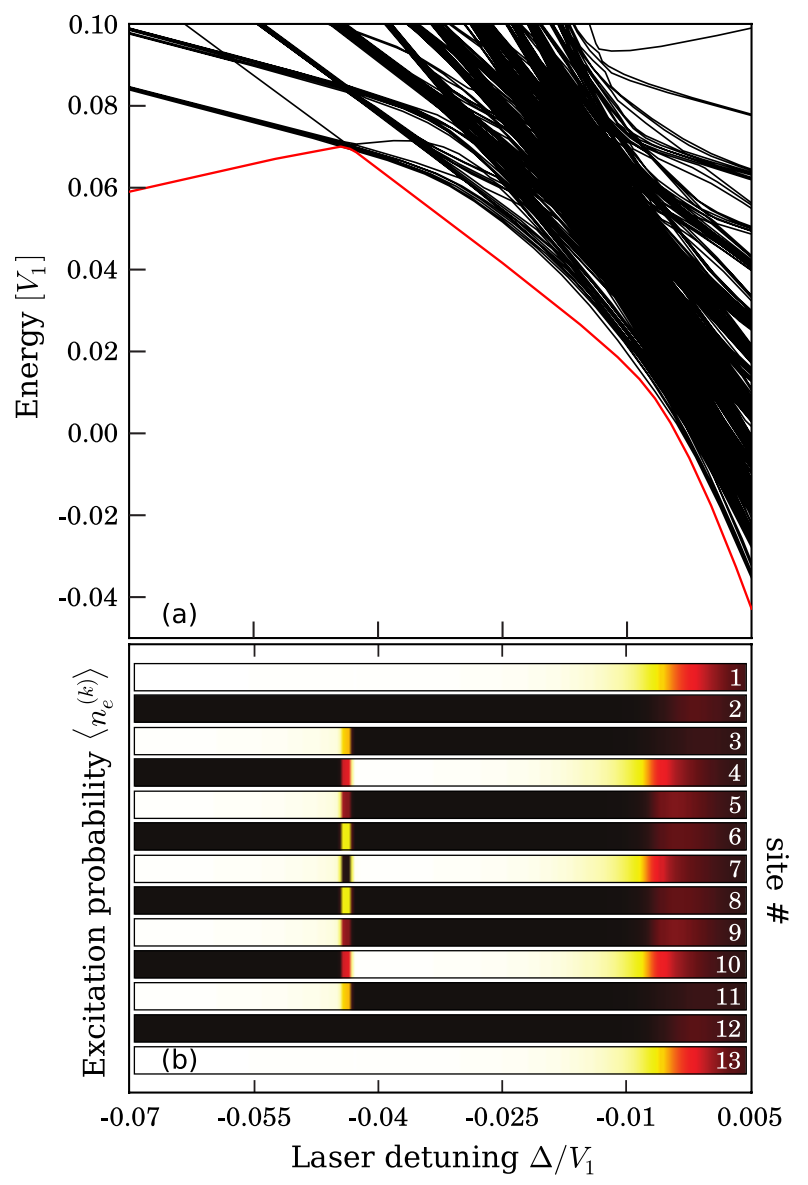

FIG. 4: (a) Detail of the energy spectrum near the ground state for $N=13$ lattice sites in the full blockade regime for small detunings and very small laser coupling $\Omega / V_{1}=0.005$. The red line indicates the ground state. At specific detunings, given by 25, avoided crossings are encountered where the ground state changes from one crystal state to another. (b) Site-resolved local excitation probability of the ground state as a function of the same laser detuning as in subfigure (a). We see from right to left a sequence of states featuring an increasing number of evenly spaced excitations. White regions correspond to unit Rydberg excitation probability, whereas dark regions denote the ground state.

one finds a succession of states with an increasing number of Rydberg excitations for increasing negative detuning. This trend continues until the completely excited lattice is encountered. Hence, the $\Delta / V_{1} \approx-2$ transition concludes what was started at the $\Delta=0$ degeneracy: a transition from the canonical product ground state to the maximally excited state with all sites occupied by Rydberg excitations. The $\Delta / V_{1} \approx-2$ transition is illustrated in figure 5 .

\section{Resonant Pair Creation}

As mentioned before, another interesting degeneracy is realized for $\Delta / V_{1}=-1 / 2$. From the degeneracy condi-

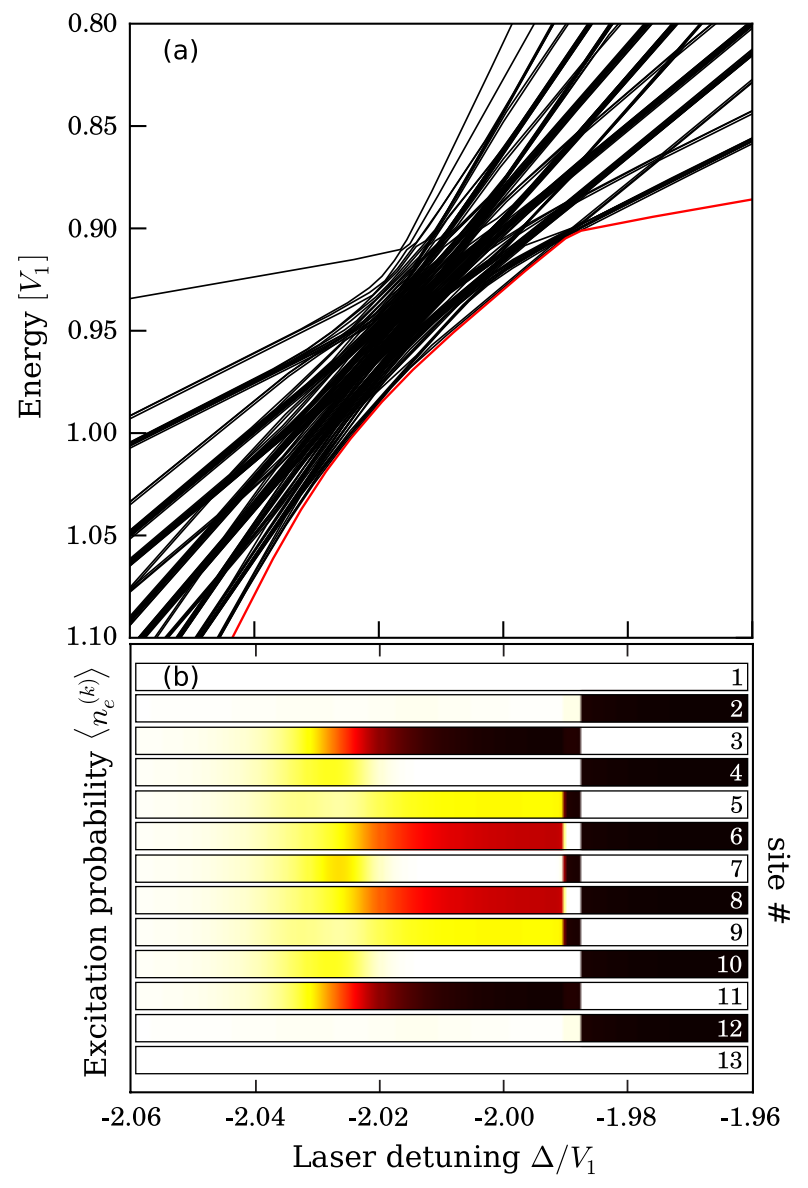

FIG. 5: Same as in figure 4 but for detunings close to $\Delta / V_{1}=-2$. One encounters the transition from the alternating excited state $|e g e \ldots g e\rangle$ (which is the final state is figure to the fully excited state $|E\rangle$.

tion (15) one finds that in this case the state $|G\rangle\left(N_{e}=\right.$ $\left.N_{e e}=0\right)$ is degenerate with all other canonical product states whose quantum numbers satisfy $N_{e}=2 N_{e e}$. Figure 6(a) presents the spectrum at the point of degeneracy for $N=13$. The maximal number of excitations compatible with both the combinatorial constraints and $N_{e}=2 N_{e e}$ is given by the maximal integer satisfying $N_{e} \leq \frac{3}{2}(N-1)$. For $N=13$ this is $N_{e}=8$ and consequently $N_{e e}=4$. Correspondingly, in subfigures 6(b) and (c) the projections $\mathcal{P}_{0,0}$ and $\mathcal{P}_{8,4}$ are shown for the two distinct states that evolve from and into the canonical ground state $|G\rangle$, respectively. $\mathcal{P}_{N_{e}, N_{e e}}$ is the projector onto the subspace $\mathcal{H}_{N_{e}, N_{e e}}$ containing $N_{e}$ Rydberg excitations and $N_{e e}$ Rydberg pairs. As expected, one finds a transition of the adiabatic eigenstates from the canonical ground state to the pair excited states when going through the region of degeneracy. At the crossing itself, further subspaces contribute to the investigated states as $\mathcal{P}_{0,0}+\mathcal{P}_{8,4} \neq 1$. The main such contribution stems from the $\mathcal{H}_{6,3}$ subspace while the $\mathcal{H}_{4,2}$ and $\mathcal{H}_{2,1}$ subspaces only contribute marginally. 


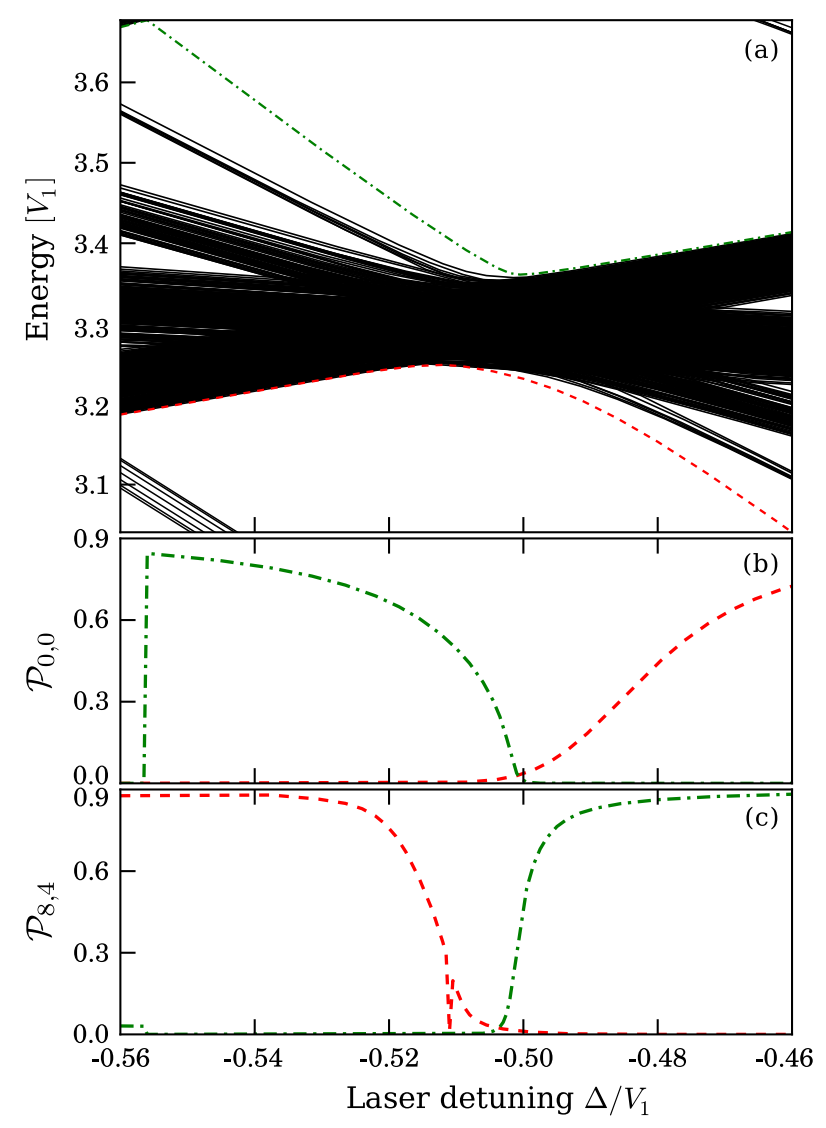

FIG. 6: (a) Detail of the energy spectrum including the canonical ground state $|G\rangle$ for detunings close to $\Delta / V_{1}=$ $-1 / 2, N=13$, and for $\Omega / V_{1}=0.1$. This specific detuning favours the excitation of Rydberg pairs. In subfigures (b) and (c) the projections $\mathcal{P}_{0,0}$ and $\mathcal{P}_{8,4}$ are shown for the two distinct states marked by the dash-dotted green and dashed red line.

\section{B. Strong Laser Regime}

As for the weak laser regime, when the laser energy contributions are very large in comparison to the nextneighbour Rydberg interactions, the system again becomes analytically treatable. Neglecting all long-range interactions, we rewrite the interaction part in this regime as

$$
\begin{aligned}
H_{\mathrm{int}} \approx & \frac{V_{1}}{4} \sum_{k=1}^{N-1} \sigma_{z}^{(k)} \sigma_{z}^{(k+1)}+\frac{V_{1}}{2} \sum_{k=1}^{N} \sigma_{z}^{(k)} \\
& -\frac{V_{1}}{4}\left[\sigma_{z}^{(1)}+\sigma_{z}^{(N)}\right]+(N-1) \frac{V_{1}}{4} .
\end{aligned}
$$

The total Hamiltonian can then be separated according to $H=H_{0}+H^{\prime}+H_{b}$ into a local part

$$
H_{0}=\frac{1}{2} \sum_{k=1}^{N}\left[\Omega \sigma_{x}^{(k)}+\left(\Delta+V_{1}\right) \sigma_{z}^{(k)}\right]+(N-1) \frac{V_{1}}{4}
$$

an interaction part $H^{\prime}=\frac{V_{1}}{4} \sum_{k=1}^{N-1} \sigma_{z}^{(k)} \sigma_{z}^{(k+1)}$, and a boundary term $H_{b}=-\frac{V_{1}}{4}\left(\sigma_{z}^{(1)}+\sigma_{z}^{(N)}\right)$. Defining the pseudo laser coupling $\tilde{\Omega}:=\sqrt{\Omega^{2}+\left(\Delta+V_{1}\right)^{2}} \gg$ $V_{1}$, the local part can be re-expressed as $H_{0}:=$ $\frac{1}{2} \tilde{\Omega} \sum_{k=1}^{N} \vec{n} \vec{\sigma}^{(k)}$ where we have introduced the notation $\vec{n} \vec{\sigma}^{(k)}=\sum_{i \in\{x, y, z\}} n_{i} \sigma_{i}^{(k)}$. The $n_{i}$ are given by

$$
\vec{n}:=\frac{1}{\tilde{\Omega}}\left(\begin{array}{c}
\Omega \\
0 \\
\Delta+V_{1}
\end{array}\right)=\left(\begin{array}{c}
\sin \theta \\
0 \\
\cos \theta
\end{array}\right) \Rightarrow \vec{n}^{2}=1 .
$$

The pseudo angle $\theta \in[0,2 \pi]$ satisfies the relation

$$
\cos \theta=\frac{\Delta+V_{1}}{\tilde{\Omega}}, \sin \theta=\frac{\Omega}{\tilde{\Omega}} .
$$

Note that $H_{0}$ contains no information about the spatial arrangement of the lattice sites. It is symmetric under any permutation of the lattice sites. Therefore, it can be diagonalized by rotating each site $k$ independently in spin-space about the $\sigma_{y}^{(k)}$ axis with the unitary transformation

$$
U_{\theta^{\prime}}:=\bigotimes_{k=1}^{N} \exp \left[-\frac{i}{2} \theta^{\prime} \sigma_{y}^{(k)}\right]
$$

It follows that

$$
U_{\theta^{\prime}}^{\dagger} \vec{n} \vec{\sigma}^{(k)} U_{\theta^{\prime}}=\cos \left(\theta-\theta^{\prime}\right) \sigma_{z}^{(k)}+\sin \left(\theta-\theta^{\prime}\right) \sigma_{x}^{(k)} .
$$

Choosing $\theta^{\prime}=\theta$, the transformed local Hamiltonian is given by

$$
\tilde{H}_{0}=U_{\theta}^{\dagger} H_{0} U_{\theta}=\frac{1}{2} \tilde{\Omega} \sum_{k=1}^{N} \sigma_{z}^{(k)}+(N-1) \frac{V_{1}}{4} .
$$

From Hamiltonian (33), the spectral features can be straightforwardly deduced. Its eigenstates are given by products of the rotated single site basis states

$$
\begin{aligned}
|+; \theta\rangle_{k} & =\cos \frac{\theta}{2}|e\rangle_{k}+\sin \frac{\theta}{2}|g\rangle_{k} \\
|-; \theta\rangle_{k} & =-\sin \frac{\theta}{2}|e\rangle_{k}+\cos \frac{\theta}{2}|g\rangle_{k}
\end{aligned}
$$

The transformed product ground state and the fully excited state $\operatorname{read}|\tilde{G} ; \theta\rangle=U_{\theta}|G\rangle=|--\cdots-; \theta\rangle$, and $|\tilde{E} ; \theta\rangle=U_{\theta}|E\rangle=|++\cdots+; \theta\rangle$, respectively. Due to the full permutation symmetry of $H_{0}$ the associated energy depends only on the number $\tilde{N}_{+}$of $|+; \theta\rangle$ factors present in a given product state,

$$
\tilde{N}_{+}:=\frac{1}{2} \sum_{k=1}^{N}\left[\sigma_{z}^{(k)}+1\right]=U_{\theta}^{\dagger} N_{+} U_{\theta}
$$




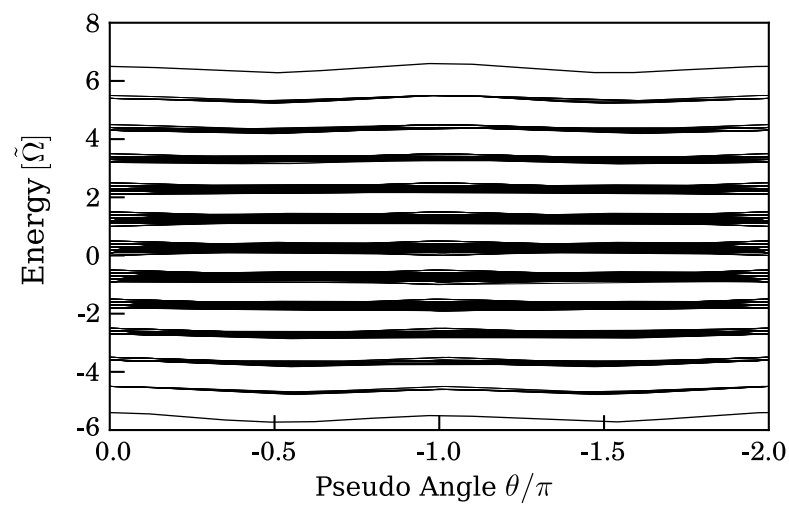

FIG. 7: Energies as a function of the pseudoangle $\theta$ for $V_{1}=0.1 \tilde{\Omega}$. The deviations from the constant energy level behaviour are strongest for $\Omega=0$ (corresponding to $\theta=$ $0, \pi, 2 \pi)$. They are always positive, which is due to the fact that the perturbing Rydberg interactions operator is strictly positive.

where $N_{+}=\frac{1}{2} \sum_{k=1}^{N}\left[\cos \theta \sigma_{z}^{(k)}+\sin \theta \sigma_{x}^{(k)}+1\right]$. The dominant part $H_{0}$ of the Hamiltonian in the strong laser coupling regime can be expressed in terms of $N_{+}$and $\tilde{N}_{+}$ as

$$
H_{0}=\tilde{\Omega}\left(N_{+}-N / 2\right)+(N-1) \frac{V_{1}}{4}
$$

and

$$
\tilde{H}_{0}=\tilde{\Omega}\left(\tilde{N}_{+}-N / 2\right)+(N-1) \frac{V_{1}}{4},
$$

respectively. The eigenvalues of $N_{+}$are $k=0,1, \ldots, N$ and for each eigenvalue $0 \leq k \leq N$ the eigenspace has dimension $\left(\begin{array}{c}N \\ k\end{array}\right)$ due to the number of possibilities to distribute $k$ excitations across a lattice of length $N$. Hence, the eigenvalues of $H_{0}$ depend only on $\tilde{\Omega}$. Moreover, its spectrum is symmetrical with respect to $E=0$ for $V_{1}=0$ and is given by

$$
E_{\kappa}=-(N-2 \kappa) \tilde{\Omega} / 2, \kappa=0,1, \ldots, N .
$$

This fact is illustrated in figure 7 where we confirm the constant spacing between the energy bands. The slight variation of the energy levels with $\theta$ stems from the Rydberg interactions which are incorporated in $H^{\prime}$ and $H_{b}$.

To understand the substructure of the energy levels at other values for $\theta$, we must focus our attention on the interaction part $H^{\prime}$. Applying the unitary transform for $\theta^{\prime}=\theta$ to the interaction Hamiltonian yields

$$
\begin{aligned}
\tilde{H}^{\prime}= & \frac{V_{1}}{4} \cos ^{2} \theta \sum_{k=1}^{N-1} \sigma_{z}^{(k)} \sigma_{z}^{(k+1)}+\frac{V_{1}}{4} \sin ^{2} \theta \sum_{k=1}^{N-1} \sigma_{x}^{(k)} \sigma_{x}^{(k+1)} \\
& -\frac{V_{1}}{4} \sin \theta \cos \theta \sum_{k=1}^{N-1}\left[\sigma_{z}^{(k)} \sigma_{x}^{(k+1)}+\sigma_{x}^{(k)} \sigma_{z}^{(k+1)}\right] .
\end{aligned}
$$

As pointed out previously, $\theta^{\prime}=\theta \approx 0, \pi$ corresponds to an almost vanishing laser intensity, i.e., we recover the weak laser regime (cf. Sec. III A). Consequently, all offdiagonal terms (i.e., those featuring at least one $\sigma_{x}^{(k)}$ operator as a factor) vanish due to the factor of $\sin \theta$. Hence, within the vicinity of these values, the energy level structure can be fully understood in terms of our previous discussion of the weak laser regime. The conceptually opposite case is realized for $\theta^{\prime}=\theta \approx \pi / 2,3 \pi / 2$. In this case the laser coupling is maximal, its magnitude being given by the pseudo laser coupling which we assume to be much larger than the Rydberg interactions and the laser detuning, $|\Omega|=\tilde{\Omega} \gg V_{1}=-\Delta$. In the following we fix $\theta=\pi / 2$; the case $\theta=3 \pi / 2$ can be treated equivalently. As mentioned before, this closely corresponds to the system analyzed in [38. The main differences are given by our requirement that $\Delta=-V_{1}$ and our open boundary conditions. Therefore we will present in the following discussion only the major differences.

For $\theta=\pi / 2$ our interaction Hamiltonian $\tilde{H}^{\prime}=$ $\frac{V_{1}}{4} \sum_{k=1}^{N-1} \sigma_{x}^{(k)} \sigma_{x}^{(k+1)}$ assumes a purely off-diagonal form. The transformed boundary term accordingly reads $\tilde{H}_{b}=$ $\frac{V_{1}}{4}\left[\sigma_{x}^{(1)}+\sigma_{x}^{(N)}\right]$. Clearly, $\tilde{H}^{\prime}$ is not diagonal in the rotated basis, but it is possible to further separate it into a part that commutes with $\tilde{H}_{0}$ and one that does not. This is achieved by rewriting $\sigma_{x}^{(k)}=\sigma_{+}^{(k)}+\sigma_{-}^{(k)}$, leading to

$$
\begin{aligned}
\tilde{H}^{\prime}= & \frac{V_{1}}{4} \sum_{k=1}^{N-1}\left[\sigma_{+}^{(k)} \sigma_{-}^{(k+1)}+\sigma_{-}^{(k)} \sigma_{+}^{(k+1)}\right] \\
& +\frac{V_{1}}{4} \sum_{k=1}^{N-1}\left[\sigma_{+}^{(k)} \sigma_{+}^{(k+1)}+\sigma_{-}^{(k)} \sigma_{-}^{(k+1)}\right] \\
\equiv & \tilde{H}_{1}^{\prime}+\tilde{H}_{2}^{\prime} .
\end{aligned}
$$

$\tilde{H}_{1}^{\prime}$ consists of operators which conserve the total number of excitations $\tilde{N}_{+}$and therefore commutes with the unperturbed Hamiltonian, i.e., $\left[\tilde{H}_{0}, \sigma_{+}^{(k)} \sigma_{-}^{(k+1)}\right]=$ $\left[\tilde{H}_{0}, \sigma_{-}^{(k)} \sigma_{+}^{(k+1)}\right]=0$ for any $k \in\{1, \ldots N-1\}$. This implies that we can find a simultaneous eigenbasis for $\tilde{H}_{0}$ and $\tilde{H}_{1}^{\prime}$. As we will show later, the remaining part of the perturbation is fully off-diagonal in this basis. Hence, for our parameter regime $\tilde{\Omega} \gg V_{1}, \Delta$ the dynamics of any initial state that belongs to a certain $\tilde{N}_{+}$eigenspace is influenced by the off-diagonal part $\tilde{H}_{2}^{\prime}$ only at second order $O\left(V_{1}^{2} / \tilde{\Omega}\right)$. Formally, this can be demonstrated by the application of quasi-degenerate perturbation theory [39. We thus neglect $\tilde{H}_{2}^{\prime}$ in the following and proceed by 
diagonalizing the resulting Hamiltonian

$$
\begin{array}{r}
\tilde{H}_{x y}:=\tilde{H}_{0}+\frac{V_{1}}{4} \sum_{k=1}^{N-1}\left[\sigma_{+}^{(k)} \sigma_{-}^{(k+1)}+\sigma_{-}^{(k)} \sigma_{+}^{(k+1)}\right] \\
=\tilde{\Omega} \sum_{k=1}^{N} \sigma_{+}^{(k)} \sigma_{-}^{(k)}-N \tilde{\Omega} / 2+(N-1) \frac{V_{1}}{4} \\
+\frac{V_{1}}{4} \sum_{k=1}^{N-1}\left[\sigma_{+}^{(k)} \sigma_{-}^{(k+1)}+\sigma_{-}^{(k)} \sigma_{+}^{(k+1)}\right] .
\end{array}
$$

As already pointed out in 31, 43] is the familiar Hamiltonian of the XY-Model [33], which can readily be diagonalized by the introduction of fermionic ladder operators

$$
c_{k}:=\sigma_{-}^{(k)} e^{i \pi \sum_{j=1}^{k-1} \sigma_{+}^{(j)} \sigma_{-}^{(j)}}=(-1)^{k-1} \sigma_{-}^{(k)} \prod_{j=1}^{k-1} \sigma_{z}^{(j)}
$$

These non-local operators and their adjoint operators obey fermionic anti-commutation rules

$$
\left\{c_{k}, c_{l}\right\}=\left\{c_{k}^{\dagger}, c_{l}^{\dagger}\right\}=0, \quad\left\{c_{k}, c_{l}^{\dagger}\right\}=\delta_{k l} .
$$

By repeated action of different $c_{k}^{\dagger}$ on the ground state $|\tilde{G}\rangle$ we can construct a basis for the state space which, by construction, is also an eigenbasis of $\tilde{N}_{+}$(and therefore $\left.\tilde{H}_{0}\right)$. It is also straightforward to confirm the following relations:

$$
\begin{aligned}
\sigma_{+}^{(k)} \sigma_{-}^{(k)} & =c_{k}^{\dagger} c_{k}, \\
\sigma_{+}^{(k)} \sigma_{-}^{(k+1)} & =c_{k}^{\dagger} c_{k+1}, \\
\sigma_{-}^{(k)} \sigma_{+}^{(k+1)} & =-c_{k} c_{k+1}^{\dagger}=c_{k+1}^{\dagger} c_{k} .
\end{aligned}
$$

Hence, the XY-Hamiltonian, which is a quadratic form in the $\sigma_{ \pm}$operators, becomes a quadratic form in the fermionic operators,

$$
\begin{aligned}
\tilde{H}_{x y}= & 2 \tilde{\Omega} \sum_{k=1}^{N} c_{k}^{\dagger} c_{k}+\frac{V_{1}}{4} \sum_{k=1}^{N-1}\left(c_{k}^{\dagger} c_{k+1}+c_{k+1}^{\dagger} c_{k}\right) \\
& -N \tilde{\Omega} / 2+(N-1) \frac{V_{1}}{4}
\end{aligned}
$$

which, by introducing the real, symmetric matrix $M=$ $\left(M_{j k}\right)_{j, k=1}^{N}$, we may rewrite as

$$
\tilde{H}_{x y}=\sum_{j=1}^{N} \sum_{k=1}^{N} c_{j}^{\dagger} M_{j k} c_{k}-N \tilde{\Omega} / 2
$$

The elements of $M$ are given by $M_{j k}=\tilde{\Omega} \delta_{j k}+\frac{V_{1}}{4}\left(\delta_{j, k+1}+\right.$ $\left.\delta_{j+1, k}\right)$. By diagonalizing $M=R^{T} \Lambda R, \Lambda_{j k}=\lambda_{j} \delta_{j k}$ with an orthogonal matrix $R$, the Hamiltonian is further simplified. This corresponds to a principal component analysis of the quadratic form in the fermionic operators.
Defining new fermionic operators $\eta_{j}:=\sum_{k=1}^{N} R_{j k} c_{k}$, the Hamiltonian decouples into $N$ independent 'fermionic' modes:

$$
\tilde{H}_{\mathrm{xy}}=\sum_{k=1}^{N} \lambda_{k} \eta_{k}^{\dagger} \eta_{k}-N \tilde{\Omega} / 2+(N-1) \frac{V_{1}}{4} .
$$

The system's ground state is still given by $|\tilde{G}\rangle=$ $|--\cdots-\rangle$ and the orthogonal transformation of the operators preserves the anti-commutator relations. Therefore, all other eigenstates can now be constructed by the successive application of $\eta_{k}^{\dagger}$ operators for different $k$ since each mode can only be occupied by a single excitation $\left[\left(\eta_{k}^{\dagger}\right)^{2}=0\right]$. The matrix elements of $R$ and the transformed operators are given by

$$
\begin{aligned}
R_{j k} & =\sqrt{\frac{2}{N+1}} \sin \left(\frac{j k \pi}{N+1}\right), \\
\eta_{j} & =\sqrt{\frac{2}{N+1}} \sum_{k=1}^{N} \sin \left(\frac{j k \pi}{N+1}\right) c_{k} .
\end{aligned}
$$

A straightforward calculation reveals that the eigenvalues of $M$ are given by $\lambda_{j}=\tilde{\Omega}+\frac{V_{1}}{2} \cos \left(\frac{j \pi}{N+1}\right)$. Hence, our resulting Hamiltonian reads

$\tilde{H}_{\mathrm{xy}}=\sum_{k=1}^{N} \eta_{k}^{\dagger} \eta_{k}\left[\tilde{\Omega}+\frac{V_{1}}{2} \cos \left(\frac{k \pi}{N+1}\right)\right]-N \tilde{\Omega} / 2+(N-1) \frac{V_{1}}{4}$.

The fermionic excitation number operators $\eta_{n}^{\dagger} \eta_{n}$ appearing in this Hamiltonian have only 0 and 1 as eigenvalues. Neglecting momentarily the term $-N \tilde{\Omega} / 2+(N-1) \frac{V_{1}}{4}$, the spectrum is thus given by

$$
\begin{aligned}
& \left\{E_{k_{1} k_{2} \ldots k_{l}}=\tilde{\Omega}\left[l+\frac{V_{1}}{2 \tilde{\Omega}} \sum_{n=1}^{l} \cos \left(\frac{k_{n} \pi}{N+1}\right)\right]\right. \\
& \left.\quad l \in\{1, \ldots, N\}, k_{1}<k_{2}<\cdots<k_{l} \in\{1,2, \ldots, N\}\right\} .
\end{aligned}
$$

The corresponding basis states are given by $\left|k_{1} k_{2} \ldots k_{l}\right\rangle:=\eta_{k_{1}}^{\dagger} \eta_{k_{2}}^{\dagger} \cdots \eta_{k_{l}}^{\dagger}|\tilde{G}\rangle$. Note that our total excitation number operator obeys

$$
\begin{aligned}
\tilde{N}_{+} & =\sum_{k=1}^{N} \sigma_{+}^{(k)} \sigma_{-}^{(k)}=\sum_{k=1}^{N} c_{k}^{\dagger} c_{k} \\
& =\sum_{k, n, m=1}^{N} R_{n k} R_{m k} \eta_{n}^{\dagger} \eta_{m}=\sum_{n=1}^{N} \eta_{n}^{\dagger} \eta_{n}
\end{aligned}
$$

and counts the $\eta$-mode excitations:

$$
\tilde{N}_{+}\left|k_{1} k_{2} \ldots k_{l}\right\rangle=l\left|k_{1} k_{2} \ldots k_{l}\right\rangle \text {. }
$$




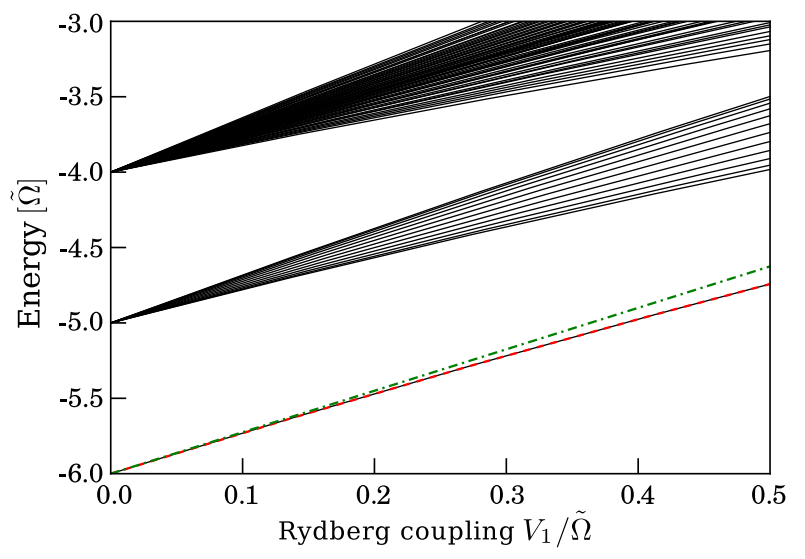

FIG. 8: Energy spectrum in the strong laser regime, showing the ground state, the $N_{+}=1$ subspace, and partially the $N_{+}=2$ subspace for $N=12$. Without the effect of the perturbation $\tilde{H}_{2}{ }_{2}$, the energy levels would scale linearly with $V_{1}$ for fixed $\tilde{\Omega}$. Here, we see that for large perturbations, the linear scaling is slightly violated. For the ground level we have also plotted the analytical result of the unperturbed energy level (green, dash-dotted line) as well as the result of second order perturbation theory (red, dashed line).

This confirms that the neglected second part of the original perturbation $\tilde{H}^{\prime}{ }_{2}=\frac{V_{1}}{4} \sum_{k=1}^{N-1}\left[\sigma_{+}^{(k)} \sigma_{+}^{(k+1)}+\right.$ $\left.\sigma_{-}^{(k)} \sigma_{-}^{(k+1)}\right]$ is fully off-diagonal in our final eigenbasis, as it maps any basis state with $l$ excitations into a superposition of states containing $l \pm 2$ excitations. Hence, for $\tilde{\Omega} \gg V_{1}$ the off-diagonal perturbation only contributes to the spectrum at second order $O\left(V_{1}^{2} / \tilde{\Omega}\right)$ and to the eigenstates at order $O\left(V_{1} / \tilde{\Omega}\right)$, justifying our former approach.

For the ground state and the first excited states we have explicitly calculated the resulting second order energy corrections (see appendix). Figure 8 presents the numerically calculated spectrum of $H$ as well as the analytically calculated results for the ground state. A comparison of the analytical and numerical results for the energy eigenvalues of the $\tilde{N}_{+}=1$ subspace $\left\{\eta_{k}^{\dagger}|G\rangle, k=\right.$ $1,2, \ldots, N\}$ is provided in table I In general, a very good agreement between the analytical and numerical results is obtained, demonstrating that $\tilde{H}_{b}$ and $\tilde{H}_{2}^{\prime}$ only lead to minor corrections.

For completeness, we give a brief discussion of the symmetry properties of the eigenstates. A detailed calculation reveals that the transformation properties under reflection are

$$
\mathcal{R}^{\dagger} \eta_{k}^{\dagger} \mathcal{R}=(-1)^{k+1} \eta_{k}^{\dagger}(-1)^{\tilde{N}_{+}}=(-1)^{\tilde{N}_{+}+k} \eta_{k}^{\dagger} .
$$

For a single fermionic excitation $\eta_{k}^{\dagger}|\tilde{G}\rangle$, its eigenvalue with respect to the reflection symmetry operator is correspondingly given by $(-1)^{k+1}$. Hence, only states corresponding to an odd $k=1,3,5, \ldots$ belong to the same symmetry eigenspace as the canonical product ground state. For a general state $\eta_{k_{1}}^{\dagger} \eta_{k_{2}}^{\dagger} \cdots \eta_{k_{l}}^{\dagger}|\tilde{G}\rangle$ the eigen-
TABLE I: Comparison of the $N_{+}=1$ energy levels calculated analytically from the unperturbed $H_{X Y}$ Hamiltonian with results obtained via second order perturbation theory and the exact numerical results for $N=12$ and $V_{1} / \tilde{\Omega}=0.1$.

\begin{tabular}{cccc}
\hline \hline$k$ & $E_{k}^{(0)}$ & $E_{k}^{(0)}+\Delta E_{k}^{(2)}$ & $E_{k}^{\text {num }}$ \\
\hline 1 & -4.676 & -4.681 & -4.681 \\
2 & -4.681 & -4.685 & -4.685 \\
3 & -4.688 & -4.691 & -4.692 \\
4 & -4.697 & -4.701 & -4.700 \\
5 & -4.707 & -4.709 & -4.711 \\
6 & -4.719 & -4.723 & -4.722 \\
7 & -4.731 & -4.733 & -4.734 \\
8 & -4.743 & -4.746 & -4.746 \\
9 & -4.753 & -4.756 & -4.757 \\
10 & -4.762 & -4.767 & -4.766 \\
11 & -4.769 & -4.774 & -4.774 \\
12 & -4.774 & -4.778 & -4.778 \\
\hline \hline
\end{tabular}

value is given by $(-1)^{k_{1}+k_{2}+\ldots k_{l}+l(l+1) / 2}$.

In concluding this section, we remark that the results obtained here only apply in a rotating frame of reference due to the RWA picture. The expectation value with respect to a given state $|\psi\rangle$ of any operator $\hat{O}$ diagonal in the canonical product basis $\mathcal{S}$ can be evaluated as usual: $\langle\hat{O}\rangle=\langle\psi|\hat{O}| \psi\rangle$. Any off-diagonal operator, on the other hand, must first be transformed to the RWA frame and therefore becomes explicitly time dependent. Within the weak laser regime discussed in the previous section, most of the relevant observables are diagonal in the canonical product basis and are thus not affected by the RWA-picture. For the strong laser regime eigenbasis, the situation is more complicated and needs to be inspected individually.

\section{Intermediate Regime}

In the intermediate regime, where our parameters $\Delta, \Omega, V_{1}$ can be of the same magnitude, we cannot separate the energy scales due to Rydberg interactions and of the laser contributions. We will therefore limit ourselves to the discussion of some numerically obtained results. As in the previous section, we fix the relative scale of Rydberg interaction energies and laser contributions by varying the pseudo angle $\theta$ for fixed $\tilde{\Omega}$ and $V_{1}$. Figure 9 provides two examples of the resulting spectrum, namely for $V_{1}=\tilde{\Omega} / 2$ and $V_{1}=2 \tilde{\Omega}$, respectively. For $\theta=0, \pi, 2 \pi$, the Hamiltonian is diagonal in the canonical product basis $\mathcal{S}_{N}$ and the spectra can be fully understood from our discussion of the weak laser regime. For intermediate values of $\theta$, however, our analytical results for the strong laser regime break down because the mixing of the energy bands due to the neglected part of the interactions and the boundary term eventually prevail. Accordingly, 


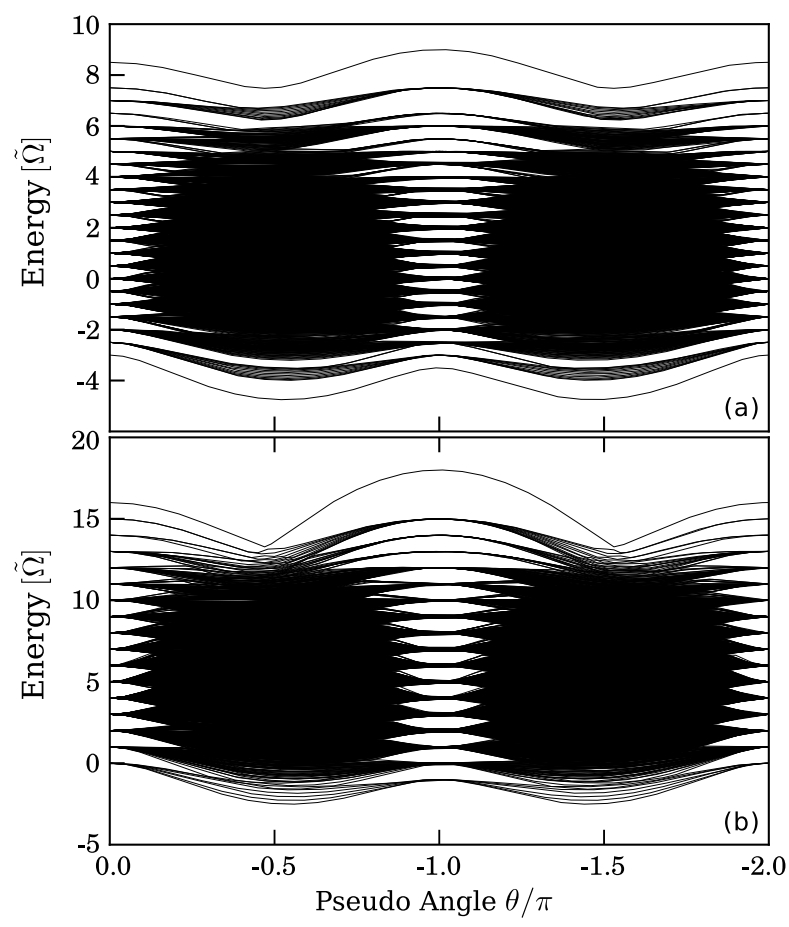

FIG. 9: Energy spectrum obtained in the intermediate regime as a function of varying $\theta$ while keeping the Rydberg interaction energy and the pseudo laser coupling constant. (a) $V_{1} / \tilde{\Omega}=1 / 2$ and (b) $V_{1} / \tilde{\Omega}=2$. The clear separation of the energy bands as seen for the strong laser regime in figure 7 is lost.

in figure 9 the corresponding energy bands cannot be resolved anymore and only the lowest and highest energy states remain well separated.

\section{NON-UNIFORM LATTICES}

In this section we generalize our setup to allow for lattices with variable lattice spacings. Due to the spatial dependence of the inter-site interactions this introduces new energy scales into the spectrum. To account for the different lattice spacings, we define the spatially dependent lattice spacing $a^{(k)}$ to be the distance between the lattice sites $k$ and $k+1$. Our interaction coefficients are then given by

$$
\mathcal{V}_{k, k+l}=\frac{C_{n}}{\left[\sum_{j=1}^{l} a^{(k+j-1)}\right]^{n}} .
$$

\section{A. Alternating Spacings}

We initially consider the specific case of a lattice with two alternating spacings $a_{1}, a_{2}$, such that the lattice sites are effectively grouped in pairs separated by the smaller spacing. For an even number of lattice sites this pre- serves the reflection symmetry of the Hamiltonian. For simplicity we introduce the ratio $\Gamma=a_{1} / a_{2}$ between the lattice spacings. Then, the next-neighbour interactions are given by $V:=C_{6} / a_{1}^{6}$ and $W:=C_{6} / a_{2}^{6}=\Gamma^{6} V$, respectively. For $\Omega / V \ll 1$ we can again realize a weak laser coupling regime that allows for an analytical investigation similar to Sec. IIIA. We split up the Hamiltonian $H=H_{0}+H_{L}+H_{\mathrm{int}}^{\mathrm{rr}}$ into a dominant contribution $H_{0}=H_{\mathrm{int}}^{\mathrm{nn}}+H_{D}$ given by the familiar laser detuning operator and the next-neighbour Rydberg interactions and a perturbation given by the comparatively small laser coupling Hamiltonian and the long range Rydberg interactions $H_{\mathrm{int}}^{\mathrm{lr}}$. For an even number of lattice sites, the next-neighbour interactions may be written as

$$
\begin{aligned}
H_{\mathrm{int}}^{\mathrm{nn}} & =V \sum_{k=1}^{N / 2} n_{e}^{(2 k-1)} n_{e}^{(2 k)}+W \sum_{k=1}^{N / 2} n_{e}^{(2 k)} n_{e}^{(2 k+1)} \\
& =V\left(N_{e e}^{V}+\Gamma N_{e e}^{W}\right) .
\end{aligned}
$$

Here, we have already divided the next-neighbour interactions $H_{\mathrm{int}}^{\mathrm{nn}}$ into pairs separated by $a_{1}$ and $a_{2}$, respectively, by introducing the corresponding next-neighbour pair operators $N_{e e}^{V}=\sum_{k=1}^{N / 2} n_{e}^{(2 k-1)} n_{e}^{(2 k)}$ and $N_{e e}^{W}=$ $\sum_{k=1}^{N / 2} n_{e}^{(2 k)} n_{e}^{(2 k+1)}$, respectively. In the above-mentioned case of a lattice with an odd number of sites, these two operators must be appropriately modified, but most of the general spectral features are similar. The unperturbed Hamiltonian $H_{0}$ is thus given by

$$
H_{0}=\Delta\left(N_{e}-N / 2\right)+V\left[N_{e e}^{V}+\Gamma^{6} N_{e e}^{W}\right] .
$$

Since $H_{0}$ is diagonal in the canonical product basis, this again leads to a simple formula for the eigenvalue of $H_{0}$ for a canonical basis state $|S\rangle \in \mathcal{S}_{\mathcal{H}}$,

$$
E^{\Gamma}(S)=\Delta\left[N_{e}(S)-N / 2\right]+V\left[N_{e e}^{V}(S)+\Gamma^{6} N_{e e}^{W}(S)\right],
$$

where $N_{e e}^{V}(S)$ and $N_{e e}^{W}(S)$ are the eigenvalues of the excitation pair operators $N_{e e}^{V}$ and $N_{e e}^{W}$ for the state $|S\rangle$.

Figure 10 presents the resulting energy spectrum and excitation pattern of the ground state obtained for $\Gamma^{6}=2$ and an odd number of lattice sites $N=13$. Comparing this result with the case of a constant spacing (equivalent to $\Gamma=1$ ) shows a qualitatively different excitation pattern for the energetic ground state for $\Delta \leq-V$. Formally, the resulting energy eigenvalues [cf. (63)] share some similarities with our result obtained for a constant lattice spacing [cf. [12]]. $V$ plays the role of $V_{1}$ while $N_{e e}^{\text {eff }}:=\left[N_{e e}^{V}(S)+\Gamma^{6} N_{e e}^{W}(S)\right]$ may be understood as an effective, in our case of $\Gamma^{6}=2$ integer, pair number. It is clear that, depending on the specific value of $\Gamma^{6}$, this generalization allows for more kinds of degeneracies as observed in figure 10(a). Although there exist further combinatorial constraints on $N_{e e}^{W}(S)$ and $N_{e e}^{V}(S)$ (e.g., $\left.N_{e e}^{W}+N_{e e}^{V}=N_{e e}\right)$ it is now possible to bring two states featuring the same number of $N_{e}$ excitations but different excitation pair numbers into degeneracy. This cannot 


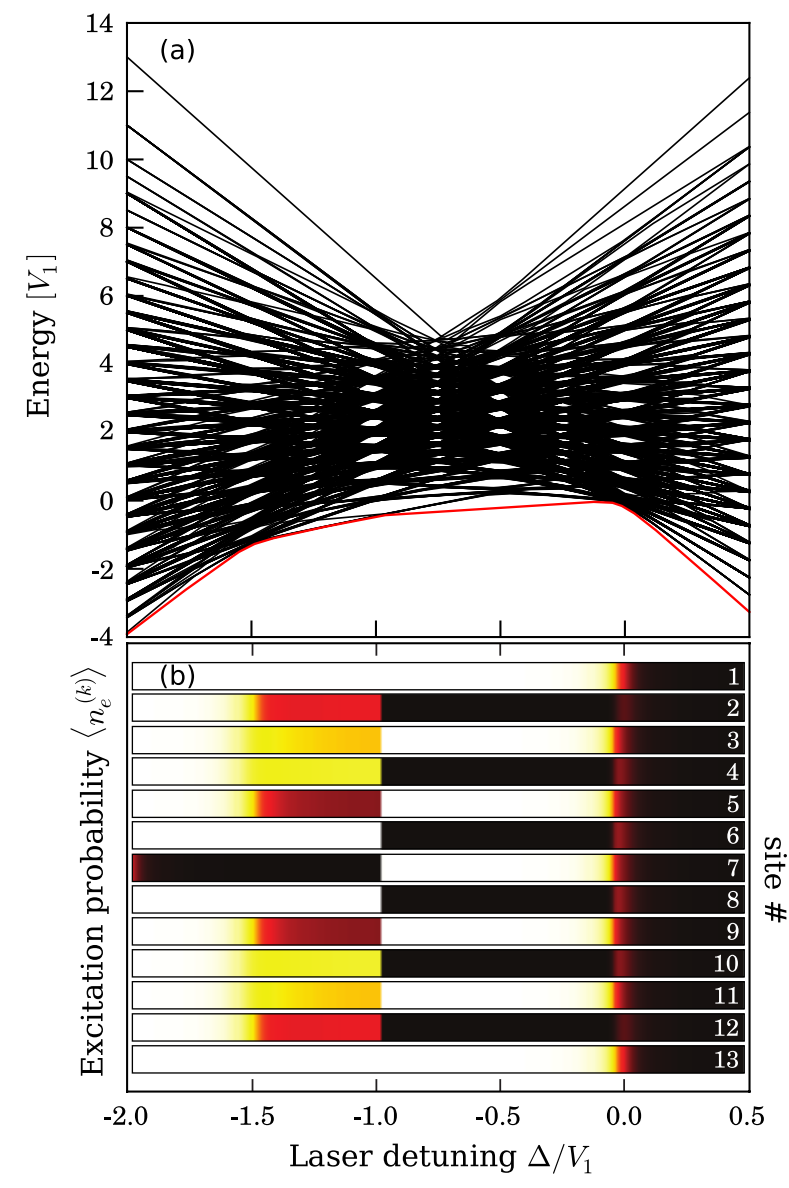

FIG. 10: (a) Spectrum and (b) local excitation probability for the energetic ground state in a patterned lattice with $N=13, \Gamma=\frac{a_{1}}{a_{2}}=\sqrt[6]{2}$ and $\Omega=0.05 \mathrm{~V}$. In contrast to the case of a single lattice spacing (cf. figure 1) we see that for $\Delta \in[-2 V,-V]$ the ground state is given by superpositions of states featuring different excitation patterns, especially for $\Delta / V<-1$. In panel (b) white regions correspond to unity Rydberg excitation probability.

be achieved dynamically, as this would require a variation of the lattice constants during the experiment, which is likely to destroy the quantum coherence of the system. But it may be exploited to adiabatically prepare superpositions of lattice states with different pair numbers $N_{e e}^{W}, N_{e e}^{V}$ by means of a time-dependent detuning $\Delta(t)$.

We also remark at this point that the results obtained so far already have an interesting consequence: distinct spectral features will depend in some fashion on the sixth power of the ratio of the lattice spacings $\Gamma^{6}$. This exponent is due to the assumed van-der-Waals interaction potential which scales with the interatomic distance $R$ as $R^{-6}$. For a general scaling $\mathcal{V}(R) \propto R^{-n}$ the ratio would enter as $\Gamma^{n}$. Hence, an experimental study of such a system could exploit this to verify the spatial dependence of the interaction potential. This would be achieved by choosing values for $\Delta, V$ and $\Gamma$ which lead to very specific

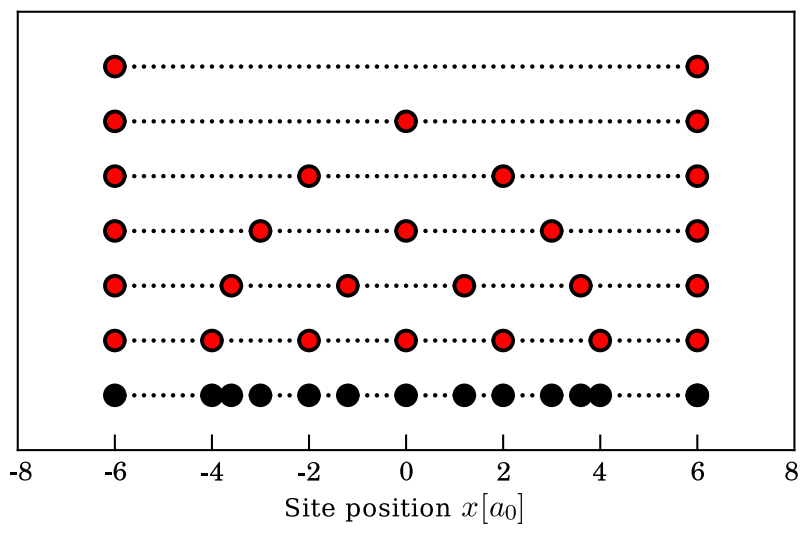

FIG. 11: The crystal states $\left|\left[N_{e}\right]\right\rangle$ (excitations marked in red) are presented for $N_{e}=2,3,4,5,6,7$ in a lattice of size $N_{0}=61$. As can be seen, to realize these states, only $N=13$ lattice sites (lowest row) must actually be occupied by atoms.

and ideally experimentally testable degeneracies. However, this goes beyond the scope of the present work and will be subject of a separate study.

\section{B. Crystal State Transitions}

A further interesting sequence of ground state transitions follows from our previous investigation of the succession of crystal states in the full blockade regime. For $N=13$ we can realize pronounced transitions $|[2]\rangle \rightarrow$ $|[3]\rangle \rightarrow|[4]\rangle \rightarrow|[5]\rangle$ for the case of a single-spaced lattice (see section III). For a lattice of size $N_{0}=61$, on the other hand, we would be able to achieve two additional transitions $|[2]\rangle \rightarrow|[3]\rangle \rightarrow|[4]\rangle \rightarrow|[5]\rangle \rightarrow|[6]\rangle \rightarrow|[7]\rangle$. However, the energy spacing between the crystal states scales with $(N-1)^{-6}$ for a fixed number of excitations, rendering an experimental realization impractical. This drawback can be compensated, though, by decreasing the lattice constant $a_{0}$.

There exists an alternative possibility of realizing the $N=61$ crystal state transitions with a strongly nonuniform lattice consisting of only $N=13$ lattice sites. In principle this can be thought of a full $N=61$ lattice, where the atoms have been removed from 48 sites. Figure 11illustrates the corresponding spatial pattern according to which the sites have to be arranged. For the case of figure 11 , the values of the detunings at the transition points are given by 25 with $N=13$, providing a more favourable scaling of the energy spacing between the crystal states. As indicated above, the same transition detunings can be achieved by a uniform $N=61$ lattice with a reduced lattice spacing of $0.2 a_{0}$. Here, $a_{0}$ denotes the spacing of a uniform lattice consisting of $N=13$ sites. The redistributed $N=13$ lattice, on the other hand, only demands a minimal spacing of $0.4 a_{0}$. If the minimal lattice spacing is experimentally limited, the redistributed $N=13$ lattice thus provides a more 


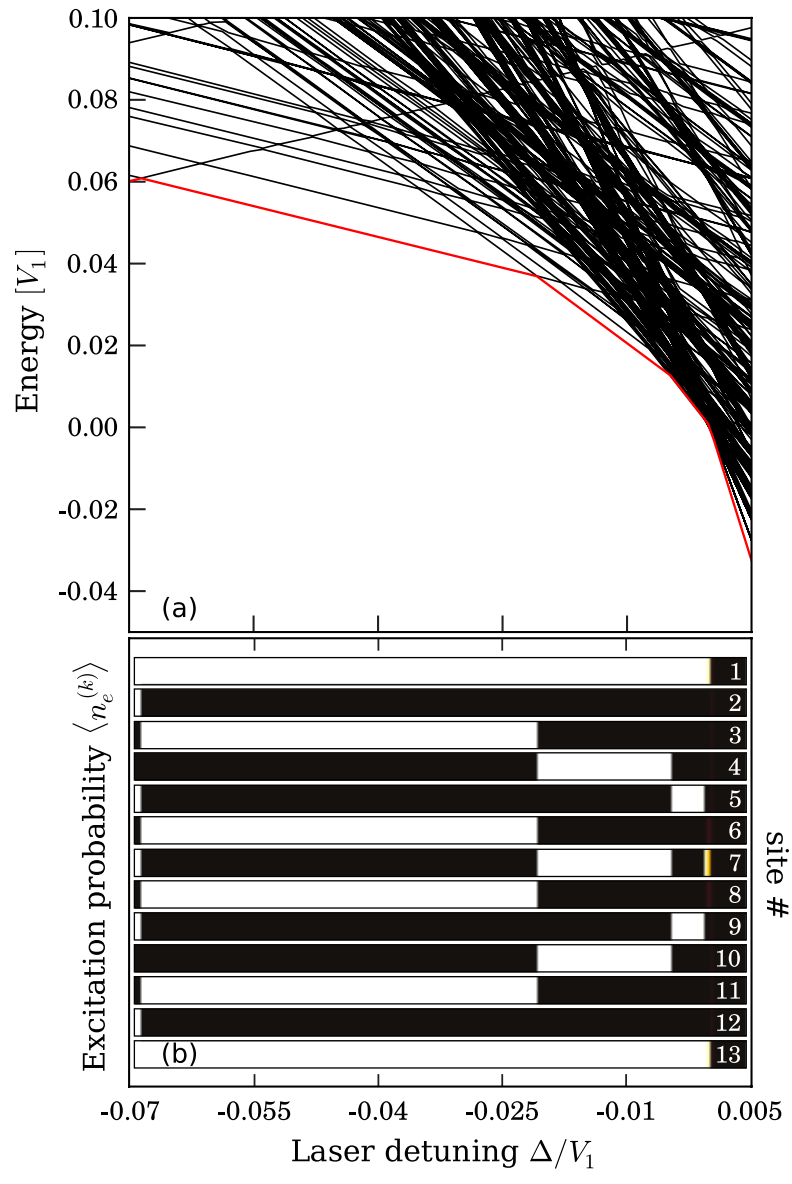

FIG. 12: (a) Spectrum and (b) local excitation probability for the energetic ground state in the patterned lattice of figure 11 with $\Omega=10^{-4} V$. As discussed in the text, this particular setup emulates a $N_{0}=61$ site lattice. The transitions between the crystal states $\left|\left[N_{e}\right]\right\rangle$ are evident in panel (b) where white regions correspond to unity Rydberg excitation probability.

favourable scaling of the relevant detunings.

Figure 12 (a) presents the energy spectrum with the first five transitions between crystal states. In panel (b) the corresponding local Rydberg excitation probability is illustrated. One can nicely observe the transitions between the crystal states. In order to get the sharp transitions between the crystal states, a relatively small Rabi frequency of $\Omega=10^{-4} V$ is assumed in this figure. Due to the sensitive scaling of the corresponding transition detuning values with the interaction exponent $n$, their exact experimental measurement could provide a useful test of the validity of the assumed interaction potential.

\section{BRIEF SUMMARY}

Focusing on the frozen gas regime at ultracold temperatures, we have provided a comprehensive analysis of the spectral properties of the coherent, laser-driven Rydberg excitation in one-dimensional ordered systems. In the weak laser regime, a systematic classification of the state space has been performed. In the so-called full blockade regime, where no neighbouring Rydberg excitations in the lattice are allowed, the transitions between crystal Rydberg lattice states were identified. Turning our investigations to the strong laser regime, an extensive analytic treatment allowed the full characterization of the spectrum by performing site-specific rotations in spin space and introducing fermionic ladder operators. An excellent agreement of our perturbative treatment and the numerically obtained spectrum has been found. Going from regularly spaced to patterned lattices, we investigated two particular examples. For alternating lattice constants, an additional energy scale is introduced that alters the spectral properties as well as the local Rydberg excitation probability of the ground state. In addition, we have highlighted a specific setup involving an irregular lattice site distribution that allows the emulation of the crystal state transitions of a more extended one-dimensional lattice.

\section{Acknowledgments}

We are very grateful to Wolfgang Zeller for both fruitful discussions and practical help concerning technical aspects of this work. We thank I. Lesanovsky, B. Olmos and T. Pohl for insightful discussions. M.M. acknowledges financial support from the German Academic Exchange Service (DAAD).

\section{Appendix A: Perturbative Energy Corrections in the Strong Laser Regime}

Here we present the formulas used for calculating the perturbative energy corrections presented in Section IIIB This is achieved by re-expressing the perturbation operators in terms of the fermionic ladder operators $\eta_{k}=\sum_{l=1}^{N} R_{k l} c_{l}$. The coefficient matrix $\left(R_{k l}\right)_{k, l=1}^{N}$ is real, symmetric and orthogonal:

$$
\begin{aligned}
& R_{k l}=R_{l k}=\sqrt{\frac{2}{N+1}} \sin \frac{k l \pi}{N+1}, \\
& \sum_{l=1}^{N} R_{k l} R_{j l}=\sum_{l=1}^{N} R_{k l} R_{l j}=\delta_{k j} .
\end{aligned}
$$

This can be proved by means of the following identities. We find that for $m \in \mathbb{Z}$

$$
\sum_{n=0}^{N} \exp \left(i \frac{n m \pi}{N+1}\right)= \begin{cases}N+1 & \text { for } m \in 2(N+1) \mathbb{Z} \\ 1+i \frac{1+\cos \left(\frac{m \pi}{N+1}\right)}{\sin \left(\frac{m \pi}{N+1}\right)} & \text { for odd } m, \\ 0 & \text { otherwise. }\end{cases}
$$


From this, it is straightforward to show that for $k, l \in$ $\{1,2, \ldots, N\}$

$$
\begin{aligned}
& \sum_{n=1}^{N} \sin \left(\frac{n k \pi}{N+1}\right) \sin \left(\frac{n l \pi}{N+1}\right) \\
= & -\frac{1}{2} \Re \sum_{n=0}^{N}\left\{\exp \left[i \frac{n(k+l) \pi}{N+1}\right]-\exp \left[i \frac{n(k-l) \pi}{N+1}\right]\right\} \\
= & \frac{N+1}{2} \delta_{k l},
\end{aligned}
$$

and one finds therefore

$$
\begin{aligned}
& \sum_{n=1}^{N} \sin \left(\frac{n k \pi}{N+1}\right) \cos \left(\frac{n l \pi}{N+1}\right) \\
= & \frac{1}{2} \Im \sum_{n=0}^{N}\left\{\exp \left[i \frac{n(k+l) \pi}{N+1}\right]+\exp \left[i \frac{n(k-l) \pi}{N+1}\right]\right\} \\
= & \begin{cases}0 & \text { for even }(k \pm l) \\
\frac{\sin \left(\frac{k \pi}{N+1}\right)}{\cos \left(\frac{l \pi}{N+1}\right)-\cos \left(\frac{k \pi}{N+1}\right)} & \text { for odd }(k \pm l)\end{cases}
\end{aligned}
$$

Where $\Re, \Im$ indicate the real and imaginary part. The unperturbed XY-Hamiltonian reads

$$
\tilde{H}_{\mathrm{xy}}=\sum_{k=1}^{N} \eta_{k}^{\dagger} \eta_{k}\left[\tilde{\Omega}+\frac{V_{1}}{2} \cos \left(\frac{k \pi}{N+1}\right)\right]+\text { const. }
$$

and the perturbations are given by the boundary term

$$
\tilde{H}_{b}=\frac{V_{1}}{4}\left[\sigma_{x}^{(1)}+\sigma_{x}^{(N)}\right]
$$

and the off-resonant couplings

$$
\tilde{H}^{\prime}{ }_{2}=\frac{V_{1}}{4} \sum_{k=1}^{N-1}\left(\sigma_{+}^{(k)} \sigma_{+}^{(k+1)}+\sigma_{-}^{(k)} \sigma_{-}^{(k+1)}\right) .
$$

The first energy manifold is spanned by the ground state $|\tilde{G}\rangle$. The first excited energy manifold is given by the set of states that contain a single excitation

$$
\left\{\eta_{k}^{\dagger}|\tilde{G}\rangle, 1 \leq k \leq N\right\} \text {. }
$$

One finds that

$$
\begin{aligned}
\tilde{H}_{b} & =\frac{V_{1}}{4}\left[c_{1}^{\dagger}+c_{1}+\left(c_{N}^{\dagger}-c_{N}\right)(-1)^{\tilde{N}_{+}}\right] \\
& =\frac{V_{1}}{4} \sum_{k=1}^{N} R_{1 k}\left\{\left[1+(-1)^{k+\tilde{N}_{+}}\right] \eta_{k}^{\dagger}+\left[1-(-1)^{k+\tilde{N}_{+}}\right] \eta_{k}\right.
\end{aligned}
$$

where we have used $R_{N k}=(-1)^{k+1} R_{1 k}$. The second part of the perturbation is

$$
\begin{aligned}
\tilde{H}^{\prime}{ }_{2} & =\frac{V_{1}}{4} \sum_{n=1}^{N-1}\left(c_{n}^{\dagger} c_{n+1}^{\dagger}-c_{n} c_{n+1}\right) \\
& =\frac{V_{1}}{4} \sum_{k, l=1}^{N}\left(\sum_{n=1}^{N-1} R_{k, n} R_{l, n+1}\right)\left(\eta_{k}^{\dagger} \eta_{l}^{\dagger}-\eta_{k} \eta_{l}\right) .
\end{aligned}
$$

Since the last term in $\mathrm{A} 5$ is antisymmetric with respect to $k$ and $l$ and they are summed over their full range, it is convenient to calculate the antisymmetrized value of the bracketed expression. We define thus

$$
\begin{aligned}
F_{k l}:= & \frac{1}{2}\left[\left(\sum_{n=1}^{N-1} R_{k, n} R_{l, n+1}\right)-(k \leftrightarrow l)\right] \\
= & \frac{1}{N+1} \sum_{n=0}^{N}\left\{\sin \left(\frac{n k \pi}{N+1}\right) \sin \left[\frac{(n+1) l \pi}{N+1}\right]-(k \leftrightarrow l)\right\} \\
= & \frac{1}{N+1} \sum_{n=0}^{N}\left\{\left[\sin \left(\frac{n k \pi}{N+1}\right) \sin \left(\frac{n l \pi}{N+1}\right) \cos \left(\frac{l \pi}{N+1}\right)\right.\right. \\
& \left.\left.+\sin \left(\frac{n k \pi}{N+1}\right) \cos \left(\frac{n l \pi}{N+1}\right) \sin \left(\frac{l \pi}{N+1}\right)\right]-(k \leftrightarrow l)\right\} \\
= & \begin{cases}0 & \text { for even }(k \pm l), \\
\frac{2}{N+1} \frac{\sin \left(\frac{k \pi}{N+1}\right) \sin \left(\frac{l \pi}{N+1}\right)}{\cos \left(\frac{l \pi}{N+1}\right)-\cos \left(\frac{k \pi}{N+1}\right)} & \text { for odd }(k \pm l) .\end{cases}
\end{aligned}
$$

The off-resonant couplings are then given by

$$
\tilde{H}_{2}^{\prime}=\frac{V_{1}}{4} \sum_{k, l=1}^{N} F_{k l}\left(\eta_{k}^{\dagger} \eta_{l}^{\dagger}-\eta_{k} \eta_{l}\right) \text {. }
$$

We thus see explicitly that the perturbation operators only couple manifolds of different numbers of fermionic excitations, specifically $\Delta \tilde{N}_{+}= \pm 1$ for $\tilde{H}_{b}$ and $\Delta \tilde{N}_{+}=$ \pm 2 for $\tilde{H}^{\prime}{ }_{2}$, respectively. This implies that all first order energy corrections vanish due to the fact that $\tilde{N}_{+}$is diagonal in our basis. In our specific case we find that we can calculate the second order contributions due to $\tilde{H}_{b}$ and $\tilde{H}^{\prime}{ }_{2}$ independently, since for any given eigenstate these operators couple to different subspaces. The relevant matrix elements for the corrections to the ground state are given by

$$
\begin{aligned}
\left\langle\tilde{G}\left|\eta_{k} \tilde{H}_{b}\right| \tilde{G}\right\rangle & =\frac{V_{1}}{4} R_{1 k}\left[1-(-1)^{k}\right], \\
\left\langle\tilde{G}\left|\eta_{k} \eta_{l} \tilde{H}_{2}^{\prime}\right| \tilde{G}\right\rangle & =-\frac{V_{1}}{2} F_{k l} .
\end{aligned}
$$

From these results and the specific form of $F_{k l}$ we see that only specific states contribute to the energy corrections. This is due to the overall symmetry under reversal of the lattice, which is preserved by the perturbations. For the corrections to the states of the first excited manifold we need the following matrix elements

$$
\begin{aligned}
& \left\langle\tilde{G}\left|\eta_{k} \eta_{l} \tilde{H}_{b} \eta_{q}^{\dagger}\right| \tilde{G}\right\rangle \\
& \quad=\frac{V_{1}}{4}\left\{R_{1 l}\left[1+(-1)^{l}\right] \delta_{k q}-R_{1 k}\left[1+(-1)^{k}\right] \delta_{l q}\right\}, \\
& \left\langle\tilde{G}\left|\eta_{k} \eta_{l} \eta_{m} \tilde{H}^{\prime}{ }_{2} \eta_{q}^{\dagger}\right| \tilde{G}\right\rangle \\
& \quad=-\frac{V_{1}}{2}\left(F_{k l} \delta_{m q}-F_{k m} \delta_{l q}+F_{l m} \delta_{k q}\right) .
\end{aligned}
$$


The ground state energy correction is thus given by

$$
\begin{aligned}
\Delta E_{\tilde{G}}^{(2)} & =\Delta E_{\tilde{G}, b}^{(2)}+\Delta E_{\tilde{G}, \text { off }}^{(2)}, \\
\Delta E_{\tilde{G}, b}^{(2)} & =-\frac{V_{1}^{2}}{4 \tilde{\Omega}} \sum_{k=1}^{\lceil N / 2\rceil} \frac{R_{1,2 k-1}^{2}}{1+\frac{V_{1}}{2 \tilde{\Omega}} \cos \frac{(2 k-1) \pi}{N+1}}, \\
\Delta E_{\tilde{G}, \text { off }}^{(2)} & =-\frac{V_{1}^{2}}{8 \tilde{\Omega}} \sum_{k=1}^{N} \sum_{l=k+1}^{N} \frac{F_{k l}^{2}}{1+\frac{V_{1}}{4 \tilde{\Omega}}\left(\cos \frac{k \pi}{N+1}+\cos \frac{l \pi}{N+1}\right)} .
\end{aligned}
$$

Before we continue by writing down the energy corrections to the singly excited states we should note that we can further approximate the energy corrections by neglecting the next to leading order contributions in the denominators:

$$
\begin{gathered}
\Delta E_{\tilde{G}, b}^{(2)} \approx-\frac{V_{1}^{2}}{4 \tilde{\Omega}} \sum_{k=1}^{\lceil N / 2\rceil} R_{1,2 k-1}^{2}, \\
\Delta E_{\tilde{G}, \text { off }}^{(2)} \approx-\frac{V_{1}^{2}}{8 \tilde{\Omega}} \sum_{k=1}^{N} \sum_{l=k+1}^{N} F_{k l}^{2} .
\end{gathered}
$$

Making the same additional approximation as above, we find for the corrections to an excited state $\eta_{q}^{\dagger}|\tilde{G}\rangle$

$$
\begin{aligned}
\Delta E_{q}^{(2)} & =\Delta E_{q, b, \tilde{G}}^{(2)}+\Delta E_{q, b}^{(2)}+\Delta E_{q, \text { off }}^{(2)} \\
\Delta E_{q, b, \tilde{G}}^{(2)} & \approx \frac{V_{1}^{2}}{4 \tilde{\Omega}} R_{1, q}^{2} \frac{1-(-1)^{q}}{2} \\
\Delta E_{q, b}^{(2)} & \approx-\frac{V_{1}^{2}}{4 \tilde{\Omega}} \sum_{\substack{k=1 \\
k \neq q}}^{N} R_{1, k}^{2} \frac{1+(-1)^{k}}{2} \\
\Delta E_{q, \mathrm{off}}^{(2)} & \approx-\frac{V_{1}^{2}}{8 \tilde{\Omega}} \sum_{k=1}^{N}\left(\sum_{l=k+1}^{N} F_{k l}^{2}-F_{k q}^{2}\right)
\end{aligned}
$$

[1] D. Jaksch, J. I. Cirac, P. Zoller, S. L. Rolston, R. Côté, and M. D. Lukin, Phys. Rev. Lett. 85, 2208 (2000).

[2] M. D. Lukin, M. Fleischhauer, R. Côté, L. M. Duan, D. Jaksch, J. I. Cirac, and P. Zoller, Phys. Rev. Lett. 87, 037901 (2001).

[3] M. Saffman, T. G. Walker, and K. Mølmer, Rev. Mod. Phys. 82, 2313 (2010).

[4] D. Tong, S. M. Farooqi, J. Stanojevic, S. Krishnan, Y. P. Zhang, R. Côté, E. E. Eyler, and P. L. Gould, Phys. Rev. Lett. 93, 063001 (2004).

[5] K. Singer, M. Reetz-Lamour, T. Amthor, L. G. Marcassa, and M. Weidemüller, Phys. Rev. Lett. 93, 163001 (2004).

[6] T. C. Liebisch, A. Reinhard, P. R. Berman, and G. Raithel, Phys. Rev. Lett. 95, 253002 (2005).

[7] T. Vogt, M. Viteau, A. Chotia, J. Zhao, D. Comparat, and P. Pillet, Phys. Rev. Lett. 99, 073002 (2007).

[8] C. S. E. van Ditzhuijzen, A. F. Koenderink, J. V. Hernández, F. Robicheaux, L. D. Noordam, and H. B. van Linden van den Heuvell, Phys. Rev. Lett. 100, 243201 (2008).

[9] E. Urban, T. A. Johnson, T. Henage, L. Isenhower, D. D. Yavuz, T. G. Walker, and M. Saffman, Nat. Phys. 5, 110 (2009).

[10] A. Gaëtan, Y. Miroshnychenko, T. Wilk, A. Chotia, M. Viteau, D. Comparat, P. Pillet, A. Browaeys, and P. Grangier, Nat. Phys. 5, 115 (2009).

[11] J. Deiglmayr, M. Reetz-Lamour, T. Amthor, S. Westermann, A. de Oliveira, and M. Weidemüller, Opt. Commun. 264, 293 (2006).

[12] R. Heidemann, U. Raitzsch, V. Bendkowsky, B. Butscher, R. Löw, L. Santos, and T. Pfau, Phys. Rev. Lett. 99, 163601 (2007).

[13] U. Raitzsch, V. Bendkowsky, R. Heidemann, B. Butscher, R. Löw, and T. Pfau, Phys. Rev. Lett. 100, 013002 (2008).
[14] K. C. Younge and G. Raithel, New J. Phys. 11, 043006 (2009).

[15] J. V. Hernández and F. Robicheaux, J. Phys. B 41, 195301 (2008).

[16] J. V. Hernández and F. Robicheaux, J. Phys. B 41, 045301 (2008).

[17] C. Ates, T. Pohl, T. Pattard, and J. M. Rost, Phys. Rev. A 76, 013413 (2007).

[18] T. Pohl, E. Demler, and M. D. Lukin, Phys. Rev. Lett. 104, 043002 (2010).

[19] C. Ates, T. Pohl, T. Pattard, and J. M. Rost, Phys. Rev. Lett. 98, 023002 (2007).

[20] T. Amthor, C. Giese, C. S. Hofmann, and M. Weidemüller, Phys. Rev. Lett. 104, 013001 (2010).

[21] J.-H. Choi, J. R. Guest, A. P. Povilus, E. Hansis, and G. Raithel, Phys. Rev. Lett. 95, 243001 (2005).

[22] I. Lesanovsky and P. Schmelcher, Phys. Rev. Lett. 95, 053001 (2005).

[23] B. Hezel, I. Lesanovsky, and P. Schmelcher, Phys. Rev. Lett. 97, 223001 (2006).

[24] M. Mayle, B. Hezel, I. Lesanovsky, and P. Schmelcher, Phys. Rev. Lett. 99, 113004 (2007).

[25] M. Mayle, I. Lesanovsky, and P. Schmelcher, Phys. Rev. A 80, 053410 (2009).

[26] K. C. Younge, B. Knuffman, S. E. Anderson, and G. Raithel, Phys. Rev. Lett. 104, 173001 (2010).

[27] K. C. Younge, S. E. Anderson, and G. Raithel, New J. Phys. 12, 023031 (2010).

[28] S. K. Dutta, J. R. Guest, D. Feldbaum, A. WalzFlannigan, and G. Raithel, Phys. Rev. Lett. 85, 5551 (2000).

[29] R. Gerritsma, S. Whitlock, T. Fernholz, H. Schlatter, J. A. Luigjes, J.-U. Thiele, J. B. Goedkoop, and R. J. C. Spreeuw, Phys. Rev. A 76, 033408 (2007).

[30] S. Whitlock, R. Gerritsma, T. Fernholz, and R. J. C. 
Spreeuw, New J. Phys. 11, 023021 (2009).

[31] B. Olmos, R. González-Férez, and I. Lesanovsky, Phys. Rev. A 79, 043419 (2009).

[32] B. Olmos, R. González-Férez, and I. Lesanovsky, Phys. Rev. Lett. 103, 185302 (2009).

[33] E. Lieb, T. Schultz, and D. Mattis, Annals of Physics 16, 407 (1961).

[34] M. O. Scully and M. Zubairy, Quantum Optics (Cambridge University Press, 1997).

[35] M. Mayle, I. Lesanovsky, and P. Schmelcher, J. Phys. B 43, 155003 (2010).
[36] K. Singer, J. Stanojevic, M. Weidemuller, and R. Cote, Journal of Physics B: Atomic, Molecular and Optical Physics 38, S295 (2005).

[37] H. Weimer and H. P. Büchler, Phys. Rev. Lett. 105, 230403 (2010).

[38] B. Olmos, R. González-Férez, and I. Lesanovsky, Phys. Rev. A 81, 023604 (2010).

[39] I. Shavitt and L. T. Redmon, J. Chem. Phys. 73, 5711 (1980). 